\title{
On two notions of complexity of algebraic numbers
}

by

\section{Yann Bugeaud (Strasbourg) and Jan-Hendrik Evertse (Leiden)}

To Wolfgang Schmidt on his seventy-fifth birthday, with all best wishes and many thanks for his mathematical inspiration

1. Introduction. We derive new, improved lower bounds for the block complexity of an irrational algebraic number and for the number of digit changes in the $b$-ary expansion of an irrational algebraic number. To this end, we apply a version of the quantitative subspace theorem by Evertse and Schlickewei [14, Theorem 2.1].

Throughout the present paper, $b$ always denotes an integer $\geq 2$ and $\xi$ is a real number with $0<\xi<1$. There exists a unique infinite sequence $\mathbf{a}=\left(a_{j}\right)_{j \geq 1}$ of integers from $\{0,1, \ldots, b-1\}$, called the $b$-ary expansion of $\xi$, such that

$$
\xi=\sum_{j \geq 1} \frac{a_{j}}{b^{j}},
$$

and a does not terminate in an infinite string of the digit $b-1$. Clearly, the sequence $\mathbf{a}$ is ultimately periodic if, and only if, $\xi$ is rational. With a slight abuse of notation, we also denote by a the infinite word $a_{1} a_{2} \ldots$ To measure the complexity of $\xi$, we measure the complexity of a. Among the different ways to do this, two notions of complexity have been recently studied. A first one, namely the block complexity, consists in counting the number $p(n, \xi, b)=p(n, \mathbf{a})$ of distinct blocks of length $n$ occurring in the word a, that is,

$$
p(n, \xi, b)=\operatorname{Card}\left\{a_{k+1} a_{k+2} \ldots a_{k+n}: k \geq 0\right\} .
$$

A second one deals with the asymptotic behaviour of the number of digit changes in a. The function nbdc, "number of digit changes", introduced

2000 Mathematics Subject Classification: 11J68, 11A63.

Key words and phrases: transcendence, Schmidt subspace theorem, combinatorics on words. 
in [8], is defined by

$$
\operatorname{nbdc}(n, \xi, b)=\operatorname{Card}\left\{1 \leq k \leq n: a_{k} \neq a_{k+1}\right\} \quad \text { for } n \geq 1 .
$$

Suppose from now on that $\xi$ is algebraic and irrational. Non-trivial lower bounds for $p(n, \xi, b)$ and $\operatorname{nbdc}(n, \xi, b)$ were obtained in $[1,8]$ by means of transcendence criteria that ultimately depend on the Schmidt subspace theorem [24] or on the quantitative Roth theorem $[23,16]$. It is known that

$$
\lim _{n \rightarrow+\infty} \frac{p(n, \xi, b)}{n}=+\infty
$$

and

$$
\operatorname{nbdc}(n, \xi, b) \geq 3(\log n)^{1+1 /(\omega(b)+4)}(\log \log n)^{-1 / 4}
$$

for every sufficiently large $n$, where $\omega(l)$ counts the number of distinct prime factors of the integer $l$.

Both (1.1) and (1.2) are very far from what can be expected if one believes that, regarding these notions of complexity, algebraic irrational numbers behave like almost all real numbers (in the sense of the Lebesgue measure). Thus, it is widely believed that the functions $n \mapsto p(n, \xi, b)$ and $n \mapsto \operatorname{nbdc}(n, \xi, b)$ should grow, respectively, exponentially in $n$ and linearly in $n$.

The main purpose of the present paper is to improve (1.2) for all $n$ and (1.1) for infinitely many $n$. Our results imply that

$$
p(n, \xi, b) \geq n(\log n)^{0.09} \quad \text { for infinitely many } n
$$

and

$$
\operatorname{nbdc}(n, \xi, b) \geq c(d)(\log n)^{3 / 2}(\log \log n)^{-1 / 2}
$$

for every sufficiently large $n$, where $c(d)$ is a constant depending only on the degree $d$ of $\xi$. In particular, we have been able to remove the dependence on $b$ in (1.2).

The new ingredient in the proof of (1.3) is the use of a quantitative version of the subspace theorem, while (1.1) was established by means of a standard qualitative version of the subspace theorem. Originally, quantitative versions of the subspace theorem were stated for a single inequality with a product of linear forms, and then the resulting upper bound for the number of subspaces depended on the number of places involved. Instead, we use a version for systems of inequalities each involving one linear form giving an upper bound for the number of subspaces independent of the number of places. In fact, for many applications, the version for systems of inequalities suffices, and it leads to much better results when many non-Archimedean places are involved.

Our paper is organized as follows. We begin by stating and discussing our result upon (1.1) in Section 2 and that upon (1.2) in Section 3. Then we state 
in Section 4 our main auxiliary tool, namely the quantitative parametric subspace theorem from [14]. We have included an improvement of the twodimensional case of the latter which is needed for our improvement upon (1.2); the proof of this improvement is included in an appendix at the end of our paper. This quantitative parametric subspace theorem is a statement about classes of twisted heights parametrized by a parameter $Q$, and one can deduce from this suitable versions of the quantitative subspace theorem, dealing with (systems of) Diophantine inequalities. In Section 5 we deduce a quantitative result for systems of inequalities (Theorem 5.1) fine-tuned for the applications in the present paper. In the particular case where we have only two unknowns we obtain a sharper quantitative version of a Ridout type theorem (Corollary 5.2). The proof of Theorem 2.1 splits in Sections 6 and 7, and that of Theorem 3.1 is given in Section 8. Finally, further applications of our results are discussed in Section 9.

\section{Block complexity of $b$-ary expansions of algebraic numbers.}

We keep the notation from the introduction. Recall that the real number $\xi$ is called normal in base $b$ if, for any positive integer $n$, each one of the $b^{n}$ words of length $n$ on the alphabet $\{0,1, \ldots, b-1\}$ occurs in the $b$-ary expansion of $\xi$ with the same frequency $1 / b^{n}$. The first explicit example of a number normal in base 10, namely the number

$$
0.1234567891011121314 \ldots \text {, }
$$

whose sequence of digits is the concatenation of the sequence of all positive integers ranged in increasing order, was given in 1933 by Champernowne [10]. It follows from the Borel-Cantelli lemma that almost all real numbers (in the sense of the Lebesgue measure) are normal in every integer base, but proving that a specific number, like $e, \pi$ or $\sqrt{2}$, is normal in some base remains a challenging open problem. However, it is believed that every real irrational algebraic number is normal in every integer base. This problem, which was first formulated by Émile Borel [7], is likely to be very difficult.

Assume from now on that $\xi$ is algebraic and irrational. In particular, the sequence $\mathbf{a}$ is not ultimately periodic. By a result of Morse and Hedlund [18, $19]$, every infinite word $\mathbf{w}$ that is not ultimately periodic satisfies $p(n, \mathbf{w}) \geq$ $n+1$ for $n \geq 1$. Consequently, $p(n, \xi, b) \geq n+1$ for every positive integer $n$. This lower bound was subsequently improved upon in 1997 by Ferenczi and Mauduit [15], who applied a non-Archimedean extension of Roth's theorem established by Ridout [21] to show (see also [4]) that

$$
\lim _{n \rightarrow+\infty}(p(n, \xi, b)-n)=+\infty \text {. }
$$

Then a new combinatorial transcendence criterion proved with the help of the Schmidt subspace theorem by Adamczewski, Bugeaud, and Luca [2] 
enabled Adamczewski and Bugeaud [1] to establish that

$$
\lim _{n \rightarrow+\infty} \frac{p(n, \xi, b)}{n}=+\infty .
$$

By combining ideas from [9] with a suitable version of the quantitative subspace theorem, we are able to prove the following concerning (2.1).

THEOREM 2.1. Let $b \geq 2$ be an integer and $\xi$ an algebraic irrational number with $0<\xi<1$. Then, for any real number $\eta$ such that $\eta<1 / 11$, we have

$$
\limsup _{n \rightarrow+\infty} \frac{p(n, \xi, b)}{n(\log n)^{\eta}}=+\infty .
$$

Ideas from [9] combined with Theorem 3.1 from [14] allow us to prove a weaker version of Theorem 2.1, namely that (2.2) holds for any $\eta$ smaller than $1 /(4 \omega(b)+15)$. The key point for removing the dependence on $b$ is the use of Theorem 5.1 below, and more precisely the fact that the exponent on $\varepsilon^{-1}$ in (5.9) does not depend of the cardinality of the set of places $S$.

We remark that Theorem 2.1 does not follow from (2.1). Indeed, there exist infinite words $\mathbf{w}$ having a complexity function $p$ satisfying

$$
\lim _{n \rightarrow+\infty} \frac{p(n, \mathbf{w})}{n}=+\infty \text { and } \lim _{n \rightarrow+\infty} \frac{p(n, \mathbf{w})}{n \log \log n}<+\infty .
$$

In particular, there exist morphic words satisfying (2.3). We refer the reader to [1] for the definition of a morphic word. An open question posed in [1] was whether the $b$-ary expansion of an irrational algebraic number can be a morphic word. Theorem 2.1 above allows us to make a small step towards a negative answer. Indeed, by a result of Pansiot [20], the complexity of a morphic word that is not ultimately periodic is either of order $n, n \log \log n$, $n \log n$, or $n^{2}$. It immediately follows from Theorem 2.1 that, regardless of the base $b$, if the $b$-ary expansion of an irrational algebraic number is generated by a morphism, then the complexity of this morphism is of order either $n \log n$ or $n^{2}$. However, by using combinatorical properties of morphic words and the transcendence criterion from [2], Albert [3], on page 59 of his thesis, was able to show a stronger result, namely that, regardless of the base $b$, if the $b$-ary expansion of an irrational algebraic number is generated by a morphism, then its complexity is of order $n^{2}$.

Note that our method yields the existence of a positive $\delta$ such that

$$
\limsup _{n \rightarrow+\infty} \frac{p(n, \xi, b)(\log \log n)^{\delta}}{n(\log n)^{1 / 11}}=+\infty .
$$

In order to avoid painful technical details, we decided not to give a proof of (2.4). 
3. Digit changes in $b$-ary expansions of algebraic numbers. Our next result is a new lower bound for the number of digit changes in $b$-ary expansions of irrational algebraic numbers.

Theorem 3.1. Let $b \geq 2$ be an integer. Let $\xi$ be an irrational, real algebraic number of degree $d$. There exist an effectively computable absolute constant $c_{1}$ and an effectively computable constant $c_{2}(\xi, b)$, depending only on $\xi$ and $b$, such that

$$
\operatorname{nbdc}(n, \xi, b) \geq c_{1} \frac{(\log n)^{3 / 2}}{(\log \log n)^{1 / 2}(\log 6 d)^{1 / 2}}
$$

for every integer $n \geq c_{2}(\xi, b)$.

Theorem 3.1 improves upon Theorem 1 from [8], where the exponent of $\log n$ depends on $b$ and tends to 1 as the number of prime factors of $b$ tends to infinity. This improvement is a consequence of the use of the two-dimensional case of Theorem 5.1 (dealing with systems of inequalities) instead of a result of Locher [16] (dealing with one inequality with a product of linear forms).

Theorem 3.1 allows us to improve upon many of the results from [8]. We restrict our attention to Section 7 from [8], that is, to the study of the gap series

$$
\xi_{\mathbf{n}, b}=\sum_{j \geq 1} b^{-n_{j}}
$$

for a given integer $b \geq 2$ and a non-decreasing sequence $\mathbf{n}=\left(n_{j}\right)_{j \geq 1}$ of positive integers. As mentioned in [8], it easily follows from Ridout's theorem [21] that the assumption

$$
\limsup _{j \rightarrow+\infty} \frac{n_{j+1}}{n_{j}}>1
$$

implies the transcendence of $\xi_{\mathbf{n}, b}$ (see e.g. Satz 7 from Schneider's monograph [25]).

In particular, for any positive real number $\varepsilon$, the real number $\xi_{\mathbf{n}, b}$ is transcendental when $n_{j}=2^{[\varepsilon j]}$, where $[\cdot]$ denotes the integer part function. A much sharper statement, that improves Corollaries 4 and 5 from [8], follows at once from Theorem 3.1.

Corollary 3.2. Let $b \geq 2$ be an integer. For any real number $\eta>2 / 3$, the sum of the series

$$
\sum_{j \geq 1} b^{-n_{j}}, \quad \text { where } n_{j}=2^{\left[j^{\eta}\right]} \text { for } j \geq 1
$$

is transcendental. 
To establish Corollary 3.2, it is enough to check that the number of positive integers $j$ such that $2^{\left[j^{\eta}\right]} \leq N$ is less than some absolute constant times $(\log N)^{1 / \eta}$, and to apply Theorem 3.1. Stronger transcendence results for the gap series $\xi_{\mathbf{n}, 2}$ follow from [5, 22], including the fact that Corollary 3.2 holds for any positive $\eta$ when $b=2$.

Further results are given in Section 9.

4. The quantitative parametric subspace theorem. We fix an algebraic closure $\overline{\mathbb{Q}}$ of $\mathbb{Q}$; all algebraic number fields occurring henceforth will be subfields of $\overline{\mathbb{Q}}$.

We introduce the necessary absolute values. The set $M_{\mathbb{Q}}$ of places of $\mathbb{Q}$ may be identified with $\{\infty\} \cup\{$ primes $\}$. We denote by $|\cdot|_{\infty}$ the ordinary (Archimedean) absolute value on $\mathbb{Q}$ and for a prime $p$ we denote by $|\cdot|_{p}$ the $p$-adic absolute value, normalized so that $|p|_{p}=p^{-1}$.

Let $\mathbb{K}$ be an algebraic number field. We denote by $M_{\mathbb{K}}$ the set of places (equivalence classes of non-trivial absolute values) of $\mathbb{K}$. The completion of $\mathbb{K}$ at a place $v$ is denoted by $\mathbb{K}_{v}$. Given a place $v \in M_{\mathbb{K}}$, we denote by $p_{v}$ the place in $M_{\mathbb{Q}}$ lying below $v$. We choose the absolute value $|\cdot|_{v}$ in $v$ in such a way that the restriction of $|\cdot|_{v}$ to $\mathbb{Q}$ is $|\cdot|_{p_{v}}$. Further, we define the normalized absolute value $\|\cdot\|_{v}$ by

$$
\|\cdot\|_{v}:=|\cdot|_{v}^{d(v)} \quad \text { where } \quad d(v):=\frac{\left[\mathbb{K}_{v}: \mathbb{Q}_{p_{v}}\right]}{[\mathbb{K}: \mathbb{Q}]} .
$$

These absolute values satisfy the product formula

$$
\prod_{v \in M_{\mathbb{K}}}\|x\|_{v}=1 \quad \text { for } x \in \mathbb{K}^{*} .
$$

Further, they satisfy the extension formula: Suppose that $\mathbb{E}$ is a finite extension of $\mathbb{K}$ and normalized absolute values $\|\cdot\|_{w}\left(w \in M_{\mathbb{E}}\right)$ are defined in precisely the same manner as those for $\mathbb{K}$. Then if $w \in M_{\mathbb{E}}$ and $v \in M_{\mathbb{K}}$ is the place below $w$, we have

$$
\|x\|_{w}=\|x\|_{v}^{d(w \mid v)} \quad \text { for } x \in \mathbb{K}, \quad \text { where } \quad d(w \mid v):=\frac{\left[\mathbb{E}_{w}: \mathbb{K}_{v}\right]}{[\mathbb{E}: \mathbb{K}]} .
$$

Notice that

$$
\sum_{w \mid v} d(w \mid v)=1
$$

where by $w \mid v$ we indicate that $w$ runs through all places of $\mathbb{E}$ lying above $v$.

Let again $\mathbb{K}$ be an algebraic number field, and $n$ an integer $\geq 2$. Let $\mathcal{L}=\left(L_{i v}: v \in M_{\mathbb{K}}, i=1, \ldots, n\right)$ be a tuple of linear forms with the following properties:

$$
L_{i v} \in \mathbb{K}\left[X_{1}, \ldots, X_{n}\right] \quad \text { for } v \in M_{\mathbb{K}}, i=1, \ldots, n,
$$


(4.5) $L_{1 v}=X_{1}, \ldots, L_{n v}=X_{n} \quad$ for all but finitely many $v \in M_{\mathbb{K}}$,

(4.6) $\quad \operatorname{det}\left(L_{1 v}, \ldots, L_{n v}\right)=1 \quad$ for $v \in M_{\mathbb{K}}$,

$$
\operatorname{Card}\left(\bigcup_{v \in M_{\mathbb{K}}}\left\{L_{1 v}, \ldots, L_{n v}\right\}\right) \leq r
$$

Further, we define

$$
\mathcal{H}=\mathcal{H}(\mathcal{L})=\prod_{v \in M_{\mathbb{K}}} \max _{1 \leq i_{1}<\cdots<i_{n} \leq s}\left\|\operatorname{det}\left(L_{i_{1}}, \ldots, L_{i_{n}}\right)\right\|_{v},
$$

where we have written $\left\{L_{1}, \ldots, L_{s}\right\}$ for $\bigcup_{v \in M_{\mathbb{K}}}\left\{L_{1 v}, \ldots, L_{n v}\right\}$.

Let $\mathbf{c}=\left(c_{i v}: v \in M_{\mathbb{K}}, i=1, \ldots, n\right)$ be a tuple of reals with the following properties:

$$
\begin{aligned}
& c_{1 v}=\cdots=c_{n v}=0 \quad \text { for all but finitely many } v \in M_{\mathbb{K}}, \\
& \sum_{v \in M_{\mathbb{K}}} \sum_{i=1}^{n} c_{i v}=0, \\
& \sum_{v \in M_{\mathbb{K}}} \max \left(c_{1 v}, \ldots, c_{n v}\right) \leq 1 .
\end{aligned}
$$

Finally, for any finite extension $\mathbb{E}$ of $\mathbb{K}$ and any place $w \in M_{\mathbb{E}}$ we define

$$
L_{i w}=L_{i v}, \quad c_{i w}=d(w \mid v) c_{i v} \quad \text { for } i=1, \ldots, n,
$$

where $v$ is the place of $M_{\mathbb{K}}$ lying below $w$ and $d(w \mid v)$ is given by (4.2).

We define a so-called twisted height $H_{Q, \mathcal{L}, \mathbf{c}}$ on $\overline{\mathbb{Q}}^{n}$ as follows. For $\mathbf{x} \in \mathbb{K}^{n}$ define

$$
H_{Q, \mathcal{L}, \mathbf{c}}(\mathbf{x}):=\prod_{v \in M_{\mathbb{K}}} \max _{1 \leq i \leq n}\left\|L_{i v}(\mathbf{x})\right\|_{v} Q^{-c_{i v}} .
$$

More generally, for $\mathbf{x} \in \overline{\mathbb{Q}}^{n}$ take any finite extension $\mathbb{E}$ of $\mathbb{K}$ with $\mathbf{x} \in \mathbb{E}^{n}$ and put

$$
H_{Q, \mathcal{L}, \mathbf{c}}(\mathbf{x}):=\prod_{w \in M_{\mathbb{E}}} \max _{1 \leq i \leq n}\left\|L_{i w}(\mathbf{x})\right\|_{w} Q^{-c_{i w}} .
$$

Using (4.12), (4.2), (4.3), and basic properties of degrees of field extensions, one easily shows that this does not depend on the choice of $\mathbb{E}$.

Proposition 4.1. Let $n$ be an integer $\geq 2$, let $\mathcal{L}=\left(L_{i v}: v \in M_{\mathbb{K}}, i=\right.$ $1, \ldots, n)$ be a tuple of linear forms satisfying (4.4)-(4.7) and $\mathbf{c}=\left(c_{i v}\right.$ : $\left.v \in M_{\mathbb{K}}, i=1, \ldots, n\right)$ a tuple of reals satisfying (4.9)-(4.11). Further, let $0<\delta \leq 1$. Then there are proper linear subspaces $T_{1}, \ldots, T_{t_{1}}$ of $\overline{\mathbb{Q}}^{n}$, all defined over $\mathbb{K}$, with

$$
t_{1}=t_{1}(n, r, \delta)= \begin{cases}4^{(n+8)^{2}} \delta^{-n-4} \log (2 r) \log \log (2 r) & \text { if } n \geq 3, \\ 2^{25} \delta^{-3} \log (2 r) \log \left(\delta^{-1} \log (2 r)\right) & \text { if } n=2\end{cases}
$$

such that the following holds: for every real $Q$ with 


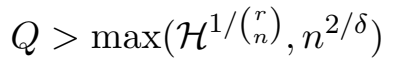

there is a subspace $T_{i} \in\left\{T_{1}, \ldots, T_{t_{1}}\right\}$ such that

$$
\left\{\mathbf{x} \in \overline{\mathbb{Q}}^{n}: H_{Q, \mathcal{L}, \mathbf{c}}(\mathbf{x}) \leq Q^{-\delta}\right\} \subset T_{i} .
$$

For $n \geq 3$ this is precisely Theorem 2.1 of [14], while for $n=2$ this is an improvement of that theorem. This improvement can be obtained by combining some lemmata from [14] with more precise computations in the case $n=2$. We give more details in the appendix at the end of the present paper.

5. Systems of inequalities. For every place $p \in M_{\mathbb{Q}}=\{\infty\} \cup\{$ primes $\}$ we choose an extension of $|\cdot|_{p}$ to $\overline{\mathbb{Q}}$ which we also denote by $|\cdot|_{p}$. For a linear form $L=\sum_{i=1}^{n} \alpha_{i} X_{i}$ with coefficients in $\overline{\mathbb{Q}}$ we define the following: We denote by $\mathbb{Q}(L)$ the field generated by the coefficients of $L$, i.e., $\mathbb{Q}(L):=$ $\mathbb{Q}\left(\alpha_{1}, \ldots, \alpha_{n}\right)$; for any map $\sigma$ from $\mathbb{Q}(L)$ to any other field we define $\sigma(L):=$ $\sum_{i=1}^{n} \sigma\left(\alpha_{i}\right) X_{i}$; and the inhomogeneous height of $L$ is given by $H^{*}(L):=$ $\prod_{v \in M_{\mathbb{K}}} \max \left(1,\left\|\alpha_{1}\right\|_{v}, \ldots,\left\|\alpha_{n}\right\|_{v}\right)$, where $\mathbb{K}$ is any number field containing $\mathbb{Q}(L)$. Further, we put $\|L\|_{v}:=\max \left(\left\|\alpha_{1}\right\|_{v}, \ldots,\left\|\alpha_{n}\right\|_{v}\right)$ for $v \in M_{\mathbb{K}}$.

Let $n$ be an integer with $n \geq 2, \varepsilon$ a real with $\varepsilon>0$ and $S=\left\{\infty, p_{1}, \ldots, p_{t}\right\}$ a finite subset of $M_{\mathbb{Q}}$ containing the infinite place. Furthermore, let $L_{i p}$ $(p \in S, i=1, \ldots, n)$ be linear forms in $X_{1}, \ldots, X_{n}$ with coefficients in $\overline{\mathbb{Q}}$ such that

$$
\begin{aligned}
& \operatorname{det}\left(L_{1 p}, \ldots, L_{n p}\right)=1 \quad \text { for } p \in S, \\
& \operatorname{Card}\left(\bigcup_{p \in S}\left\{L_{1 p}, \ldots, L_{n p}\right\}\right) \leq R, \\
& {\left[\mathbb{Q}\left(L_{i p}\right): \mathbb{Q}\right] \leq D \quad \text { for } p \in S, i=1, \ldots, n,} \\
& H^{*}\left(L_{i p}\right) \leq H \quad \text { for } p \in S, i=1, \ldots, n,
\end{aligned}
$$

and $e_{i p}(p \in S, i=1, \ldots, n)$ be reals satisfying

$$
\begin{aligned}
& e_{i \infty} \leq 1(i=1, \ldots, n), \quad e_{i p} \leq 0(p \in S \backslash\{\infty\}, i=1, \ldots, n), \\
& \sum_{p \in S} \sum_{i=1}^{n} e_{i p}=-\varepsilon .
\end{aligned}
$$

Finally, let $\Psi$ be a function from $\mathbb{Z}^{n}$ to $\mathbb{R}_{\geq 0}$. We consider the system of inequalities

$$
\left|L_{i p}(\mathbf{x})\right|_{p} \leq \Psi(\mathbf{x})^{e_{i p}} \quad(p \in S, i=1, \ldots, n) \quad \text { in } \mathbf{x} \in \mathbb{Z}^{n} \text { with } \Psi(\mathbf{x}) \neq 0 .
$$

THEOREM 5.1. The set of solutions of (5.7) with

$$
\Psi(\mathbf{x})>\max \left(2 H, n^{2 n / \varepsilon}\right)
$$

is contained in the union of at most 


$$
\begin{cases}8^{(n+9)^{2}}\left(1+\varepsilon^{-1}\right)^{n+4} \log (2 R D) \log \log (2 R D) & \text { if } n \geq 3 \\ 2^{32}\left(1+\varepsilon^{-1}\right)^{3} \log (2 R D) \log \left(\left(1+\varepsilon^{-1}\right) \log (2 R D)\right) & \text { if } n=2\end{cases}
$$

proper linear subspaces of $\mathbb{Q}^{n}$.

REMARK. Let $\|\cdot\|$ be any vector norm on $\mathbb{Z}^{n}$. Then for the solutions $\mathbf{x}$ of (5.7) we have, in view of (5.5),

$$
\|\mathbf{x}\| \ll \max _{1 \leq i \leq n}\left|L_{i \infty}(\mathbf{x})\right| \ll \Psi(\mathbf{x}) .
$$

So it would not have been a substantial restriction if in the formulation of Theorem 5.1 we had restricted the function $\Psi$ to vector norms. But for applications it is convenient to allow other functions for $\Psi$.

We deduce from Theorem 5.1 a quantitative Ridout type theorem. Let $S_{1}, S_{2}$ be finite, possibly empty sets of prime numbers, put $S:=\{\infty\} \cup S_{1} \cup$ $S_{2}$, let $\xi \in \overline{\mathbb{Q}}$ be an algebraic number, let $\varepsilon>0$, and let $f_{p}(p \in S)$ be reals such that

$$
f_{p} \geq 0 \quad \text { for } p \in S, \quad \sum_{p \in S} f_{p}=2+\varepsilon .
$$

We consider the system of inequalities

$$
\left\{\begin{array}{l}
|\xi-x / y| \leq y^{-f_{\infty}} \\
|x|_{p} \leq y^{-f_{p}} \quad\left(p \in S_{1}\right) \\
|y|_{p} \leq y^{-f_{p}} \quad\left(p \in S_{2}\right)
\end{array}\right\} \quad \text { in }(x, y) \in \mathbb{Z}^{2} \text { with } y>0 .
$$

Define the height of $\xi$ by $H(\xi):=\prod_{v \in M_{\mathbb{K}}} \max \left(1,\|\xi\|_{v}\right)$, where $\mathbb{K}$ is any algebraic number field with $\xi \in \mathbb{K}$. Suppose that $\xi$ has degree $d$.

COROllary 5.2. The set of solutions of (5.11) with

$$
y>\max \left(2 H(\xi), 2^{4 / \varepsilon}\right)
$$

is contained in the union of at most

$$
2^{32}\left(1+\varepsilon^{-1}\right)^{3} \log (6 d) \log \left(\left(1+\varepsilon^{-1}\right) \log (6 d)\right)
$$

one-dimensional linear subspaces of $\mathbb{Q}^{2}$.

To obtain Corollary 5.2 one simply has to apply Theorem 5.1 with $n=2$, $S=\{\infty\} \cup S_{1} \cup S_{2}$ and with

$$
\begin{aligned}
& L_{1 \infty}=X_{1}-\xi X_{2}, \quad L_{2 \infty}=X_{2}, \\
& L_{1 p}=X_{1}, \quad L_{2 p}=X_{2} \quad \text { for } p \in S_{1} \cup S_{2}, \\
& e_{1 \infty}=1-f_{\infty}, \quad e_{2 \infty}=1, \\
& e_{1 p}=-f_{p}, \quad e_{2 p}=0 \quad \text { for } p \in S_{1}, \\
& e_{1 p}=0, \quad e_{2 p}=-f_{p} \quad \text { for } p \in S_{2}, \\
& \Psi(\mathbf{x})=\left|x_{2}\right| \quad \text { for } \mathbf{x}=\left(x_{1}, x_{2}\right) \in \mathbb{Z}^{2} .
\end{aligned}
$$


It is straightforward to verify that (5.1) is satisfied, and that (5.2)-(5.4) are satisfied with $R=3, D=d, H=H(\xi)$, respectively. Further, it follows at once from (5.10) that (5.5) and (5.6) are satisfied.

Proof of Theorem 5.1. Let $\mathbb{K}$ be a finite normal extension of $\mathbb{Q}$, containing the coefficients of $L_{i p}$ as well as the conjugates over $\mathbb{Q}$ of those coefficients, for $p \in S, i=1, \ldots, n$. For $v \in M_{\mathbb{K}}$ we put $d(v):=\left[\mathbb{K}_{v}: \mathbb{Q}_{p_{v}}\right]$ where $p_{v}$ is the place of $\mathbb{Q}$ below $v$, and

$$
s(v)= \begin{cases}d(v) & \text { if } v \text { is Archimedean } \\ 0 & \text { if } v \text { is non-Archimedean }\end{cases}
$$

Recall that every $|\cdot|_{p}\left(p \in M_{\mathbb{Q}}\right)$ has been extended to $\overline{\mathbb{Q}}$ so in particular to $\mathbb{K}$. For every $v \in M_{\mathbb{K}}$ there is an automorphism $\sigma_{v}$ of $\mathbb{K}$ such that $\left|\sigma_{v}(\cdot)\right|_{p}$ represents $v$. So by (4.1) we have

$$
\|x\|_{v}=\left|\sigma_{v}(x)\right|_{p_{v}}^{d(v)} \quad \text { for } x \in \mathbb{K} .
$$

Let $T$ denote the set of places of $\mathbb{K}$ lying above the places in $S$. Define linear forms $L_{i v}$ and reals $e_{i v}\left(v \in M_{\mathbb{K}}, i=1, \ldots, n\right)$ by

$$
L_{i v}=\sigma_{v}^{-1}\left(L_{i, p_{v}}\right)(v \in T), \quad L_{i v}=X_{i}\left(v \in M_{\mathbb{K}} \backslash T\right)
$$

and

$$
e_{i v}=d(v) e_{i, p_{v}}(v \in T), \quad e_{i v}=0\left(v \in M_{\mathbb{K}} \backslash T\right),
$$

respectively. Then system (5.7) can be rewritten as

$$
\begin{array}{r}
\left\|L_{i v}(\mathbf{x})\right\|_{v} \leq \Psi(\mathbf{x})^{e_{i v}} \quad\left(v \in M_{\mathbb{K}}, i=1, \ldots, n\right) \\
\text { in } \mathbf{x} \in \mathbb{Z}^{n} \text { with } \Psi(\mathbf{x}) \neq 0 .
\end{array}
$$

Notice that in view of $(5.17),(5.5),(5.6)$, and $\sum_{v \mid p} d(v)=1$ for $p \in M_{\mathbb{Q}}$ we have

$$
e_{i v} \leq s(v)(i=1, \ldots, n), \quad \sum_{v \in M_{\mathbb{K}}} \sum_{i=1}^{n} e_{i v} \leq-\varepsilon .
$$

Further, by (5.2), (5.15),

$$
\operatorname{Card}\left(\bigcup_{v \in M_{\mathbb{K}}}\left\{L_{1 v}, \ldots, L_{n v}\right\}\right) \leq r:=n+D R .
$$

Now define

$$
\delta:=\frac{\varepsilon}{n+\varepsilon},
$$

let $\mathcal{L}=\left(L_{i v}: v \in M_{\mathbb{K}}, i=1, \ldots, n\right)$, and define the tuple of reals $\mathbf{c}=\left(c_{i v}\right.$ : $\left.v \in M_{\mathbb{K}}, i=1, \ldots, n\right)$ by

$$
c_{i v}:=(1+(\varepsilon / n))^{-1}\left(e_{i v}-\frac{1}{n} \sum_{j=1}^{n} e_{j v}\right) .
$$


Let $\mathcal{H}=\mathcal{H}(\mathcal{L})$ be the quantity defined by $(4.8)$ and $H_{Q, \mathcal{L}, \mathbf{c}}$ the twisted height defined by (4.13). We want to apply Proposition 4.1, and to this end we have to verify the conditions (4.4)-(4.7) and (4.9)-(4.11). Condition (4.4) is obvious. (5.1) and (5.15) imply (4.5), (4.6), while (4.7) is (5.18). Condition (4.9) is satisfied in view of (5.16), (5.20), while (4.10) follows at once from (5.21). To verify (4.11), observe that by (5.21) and (5.18) we have

$$
\begin{aligned}
\sum_{v \in M_{\mathbb{K}}} \max \left(c_{1 v}, \ldots, c_{n v}\right) & \leq\left(1+\frac{\varepsilon}{n}\right)^{-1}\left(\sum_{v \in M_{\mathbb{K}}} s(v)-\frac{1}{n} \sum_{v \in M_{\mathbb{K}}} \sum_{j=1}^{n} e_{j v}\right) \\
& =\left(1+\frac{\varepsilon}{n}\right)^{-1}\left(1+\frac{\varepsilon}{n}\right)=1 .
\end{aligned}
$$

The following lemma connects system (5.7) to Proposition 4.1.

Lemma 5.3. Let $\mathbf{x}$ be a solution of (5.7) with (5.8). Put

$$
Q:=\Psi(\mathbf{x})^{1+\varepsilon / n} \text {. }
$$

Then

$$
\begin{aligned}
& H_{Q, \mathcal{L}, \mathbf{c}}(\mathbf{x}) \leq Q^{-\delta}, \\
& Q \geq \max \left(\mathcal{H}^{\left.1 /\left(\begin{array}{l}
r \\
n
\end{array}\right), n^{2 / \delta}\right) .}\right.
\end{aligned}
$$

Proof. As observed above, $\mathbf{x}$ satisfies (5.17). In combination with (5.21) this yields

$$
\left\|L_{i v}(\mathbf{x})\right\|_{v} Q^{-c_{i v}}=\left\|L_{i v}(\mathbf{x})\right\|_{v} \cdot \Psi(\mathbf{x})^{-e_{i v}} \cdot \Psi(\mathbf{x})^{n^{-1} \sum_{j=1}^{n} e_{j v}} \leq \Psi(\mathbf{x})^{n^{-1} \sum_{j=1}^{n} e_{j v}}
$$

for $v \in M_{\mathbb{K}}, i=1, \ldots, n$. By taking the product over $v \in M_{\mathbb{K}}$ and using (5.18), (5.20) we obtain

$$
H_{Q, \mathcal{L}, \mathbf{c}}(\mathbf{x})=\prod_{v \in M_{\mathbb{K}}} \max _{1 \leq i \leq n}\left\|L_{i v}(\mathbf{x})\right\|_{v} Q^{-c_{i v}} \leq \Psi(\mathbf{x})^{-\varepsilon / n}=Q^{-\delta} .
$$

This proves (5.22).

To prove (5.23), write $\bigcup_{v \in M_{\mathbb{K}}}\left\{L_{1 v}, \ldots, L_{n v}\right\}=\left\{L_{1}, \ldots, L_{s}\right\}$. Then $s \leq r$ by (5.19). By (5.4), (5.15) we have $H^{*}\left(L_{i v}\right) \leq H$ for $v \in M_{\mathbb{K}}, i=1, \ldots, n$. By applying e.g., Hadamard's inequality for the Archimedean places and the ultrametric inequality for the non-Archimedean places, we obtain for $i_{1}, \ldots, i_{n} \in\{1, \ldots, s\}, v \in M_{\mathbb{K}}$,

$$
\left\|\operatorname{det}\left(L_{i_{1}}, \ldots, L_{i_{n}}\right)\right\|_{v} \leq\left(n^{n / 2}\right)^{s(v)} \prod_{j=1}^{n}\left\|L_{i_{j}}\right\|_{v} \leq\left(n^{n / 2}\right)^{s(v)} \prod_{i=1}^{s} \max \left(1,\left\|L_{i}\right\|_{v}\right),
$$

hence

$$
\mathcal{H} \leq n^{n / 2} \prod_{i=1}^{r} H^{*}\left(L_{i}\right) \leq n^{n / 2} H^{r}
$$


Together with (5.19), (5.20) this implies $\max \left(\mathcal{H}^{1 /\left(\begin{array}{l}r \\ n\end{array}\right)}, n^{2 / \delta}\right) \leq \max \left(n^{n / 2\left(\begin{array}{l}r \\ n\end{array}\right)} H^{r /\left(\begin{array}{l}r \\ n\end{array}\right)}, n^{2(n+\varepsilon) / \varepsilon}\right) \leq \max \left(2 H, n^{2 n / \varepsilon}\right)^{1+\varepsilon / n}$. So if $\mathbf{x}$ satisfies (5.8), then $Q=\Psi(\mathbf{x})^{1+\varepsilon / n}$ satisfies (5.23).

We apply Proposition 4.1 with the values of $r, \delta$ given by (5.19), (5.20), i.e., $r=n+D R$ and $\delta=\varepsilon /(n+\varepsilon)$. It is straightforward to show that for these choices of $r, \delta$ the quantity $t_{1}$ from Proposition 4.1 is bounded above by the quantity in (5.9). By Proposition 4.1, there are proper linear subspaces $T_{1}, \ldots, T_{t_{1}}$ of $\overline{\mathbb{Q}}^{n}$ such that for every $Q$ with (5.23) there is $T_{i} \in\left\{T_{1}, \ldots, T_{t_{1}}\right\}$ with

$$
\left\{\mathbf{x} \in \overline{\mathbb{Q}}^{n}: H_{Q, \mathcal{L}, \mathbf{c}}(\mathbf{x}) \leq Q^{-\delta}\right\} \subset T_{i} .
$$

Now Lemma 5.3 implies that the solutions $\mathbf{x}$ of (5.7) with (5.8) lie in $\bigcup_{i=1}^{t_{1}}\left(T_{i} \cap \mathbb{Q}^{n}\right)$. Theorem 5.1 follows.

6. A combinatorial lemma for the proof of Theorem 2.1. In this section, we establish the following lemma.

Lemma 6.1. Let $b \geq 2$ be an integer. Let $c$ and $u$ be positive real numbers. Let $\xi$ be an irrational real number such that $0<\xi<1$ and

$$
p(n, \xi, b) \leq c n(\log n)^{u} \quad \text { for } n \geq 1 .
$$

Then for every positive real number $v<u$, there exist integer sequences $\left(r_{n}\right)_{n \geq 1},\left(t_{n}\right)_{n \geq 1},\left(p_{n}\right)_{n \geq 1}$ and a positive real number $C$ depending only on $c, u, v$ such that

$$
\begin{gathered}
\quad\left|b^{t_{n}} \xi-b^{r_{n}} \xi-p_{n}\right| \leq\left(b^{t_{n}}\right)^{-\left(\log t_{n}\right)^{-v}} \\
0 \leq r_{n}<t_{n}, \quad 2 t_{n}<t_{n+1}, \quad t_{n} \leq(2 n)^{C n} \quad \text { for } n \geq 1 .
\end{gathered}
$$

Furthermore, $b$ does not divide $p_{n}$ if $r_{n} \geq 1$.

Proof. Let $b$ and $\xi$ be as in the statement of the lemma. Let a denote the $b$-ary expansion of $\xi$. Throughout this proof, $c_{1}, c_{2}, \ldots$ are positive constants depending only on $c, u, v$. The length of a finite word $W$, that is, the number of letters composing $W$, is denoted by $|W|$. The infinite word $W^{\infty}$ is obtained by concatenation of infinitely many copies of the finite word $W$.

By assumption, the complexity function of a satisfies

$$
p(n, \mathbf{a}) \leq c_{1} n(\log n)^{u} \quad \text { for } n \geq 1 .
$$

Our aim is to show that there exists a "dense" (in some sense) sequence of rational approximations to $\xi$ with special properties.

Let $l \geq 2$ be an integer, and denote by $A(l)$ the prefix of $\mathbf{a}$ of length $l$. By the Schubfachprinzip, there exist (possibly empty) words $U_{l}, V_{l}, W_{l}$ and $X_{l}$ such that

$$
A(l)=U_{l} V_{l} W_{l} V_{l} X_{l} \quad \text { and } \quad\left|V_{l}\right| \geq c_{2} l(\log l)^{-u} .
$$


Set $r_{l}=\left|U_{l}\right|$ and $s_{l}=\left|V_{l} W_{l}\right|$. We choose the words $U_{l}, V_{l}, W_{l}$ and $X_{l}$ in such a way that $\left|V_{l}\right|$ is maximal and, among the corresponding factorizations of $A(l)$, such that $\left|U_{l}\right|$ is minimal. In particular, either $U_{l}$ is the empty word, or the last digits of $U_{l}$ and $V_{l} W_{l}$ are different.

If $\xi_{l}$ denotes the rational number with $b$-ary expansion $U_{l}\left(V_{l} W_{l}\right)^{\infty}$, then there exists an integer $p_{l}$ such that

$$
\xi_{l}=\frac{p_{l}}{b^{r_{l}}\left(b^{s_{l}}-1\right)}, \quad\left|\xi-\xi_{l}\right| \leq b^{-r_{l}-s_{l}-\left|V_{l}\right|},
$$

and $b$ does not divide $p_{l}$ if $r_{l} \geq 1$.

Take $t_{l}=r_{l}+s_{l}$. Then

$$
l \geq t_{l} \geq s_{l} \geq c_{2} l(\log l)^{-u} .
$$

Hence,

$$
\left|b^{t_{l}} \xi-b^{r_{l}} \xi-p_{l}\right| \leq b^{-c_{2} l(\log l)^{-u}} \leq\left(b^{t_{l}}\right)^{-c_{3}\left(\log t_{l}\right)^{-u}} .
$$

We construct a sequence of positive integers $\left(l_{k}\right)_{k=1}^{\infty}$ such that for every $k \geq 1$,

$$
\begin{aligned}
& \left|b^{t_{l_{k}}} \xi-b^{r_{l_{k}}} \xi-p_{l_{k}}\right| \leq\left(b^{t_{l_{k}}}\right)^{-\left(\log t_{l_{k}}\right)^{-v}}, \\
& t_{l_{k+1}}>2 t_{l_{k}}, \\
& t_{l_{k}} \leq(2 k)^{C k} .
\end{aligned}
$$

Then a slight change of notation establishes the lemma.

Let $l_{1}$ be the smallest positive integer $l$ such that $c_{3}\left(\log t_{l}\right)^{u} \geq\left(\log t_{l}\right)^{v}$. Further, for $k=1,2, \ldots$, let $l_{k+1}$ be the smallest positive integer $l$ such that $t_{l}>2 t_{l_{k}}$. This sequence is well-defined by (6.2). It is clear that (6.3), (6.4) are satisfied.

To prove (6.5), observe that if $l$ is any integer with $c_{2} l(\log l)^{-u}>2 l_{k}$ then, by (6.2), $t_{l}>2 l_{k} \geq 2 t_{l_{k}}$. This shows that there is a constant $c_{4}$ such that $l_{k+1} \leq c_{4} l_{k}\left(\log l_{k}\right)^{u}$. Now an easy induction shows that there exists a constant $C$, depending only on $c, u, v$, such that $l_{k} \leq(2 k)^{C k}$ for $k \geq 1$. Invoking again (6.2) we obtain (6.5).

7. Completion of the proof of Theorem 2.1. Let $\xi$ be an algebraic irrational real number. Let $v$ be a real number such that $0<v<1 / 11$. Define the positive real number $\eta$ by

$$
(11+2 \eta)(v+\eta)+\eta=1 .
$$

We assume that there exists a positive constant $c$ such that the complexity function of $\xi$ in base $b$ satisfies

$$
p(n, \xi, b) \leq c n(\log n)^{v+\eta} \quad \text { for } n \geq 1,
$$

and we will derive a contradiction. Then Theorem 2.1 follows. 
Let $N$ be a sufficiently large integer. We will often use the fact that $N$ is large, in order to absorb numerical constants.

Let $\left(r_{n}\right)_{n \geq 1},\left(t_{n}\right)_{n \geq 1}$, and $\left(p_{n}\right)_{n \geq 1}$ be the sequences given by Lemma 6.1 applied with $u:=v+\eta$. Set

$$
\varepsilon=\left(\log t_{N}\right)^{-v},
$$

and observe that, in view of (6.1) and (7.3), we have

$$
\varepsilon^{-1}=\left(\log t_{N}\right)^{v} \leq N^{v+\eta} .
$$

For $n=1, \ldots, N$, we have

$$
\left|b^{t_{n}} \xi-b^{r_{n}} \xi-p_{n}\right|<\left(b^{t_{n}}\right)^{-\varepsilon} .
$$

Put

$$
k:=[2 / \varepsilon]+1 .
$$

For each $n=1, \ldots, N$ there is $l \in\{0,1, \ldots, k-1\}$ such that

$$
\frac{l}{k} \leq \frac{r_{n}}{t_{n}}<\frac{l+1}{k} \text {. }
$$

For the moment, we consider those $n \in\{1, \ldots, N\}$ such that

$$
\frac{N}{2} \leq n \leq N, \quad \frac{l}{k} \leq \frac{r_{n}}{t_{n}}<\frac{l+1}{k},
$$

where $l \in\{0,1, \ldots, k-1\}$ is fixed, and show that the vectors

$$
\mathbf{x}_{n}:=\left(b^{t_{n}}, b^{r_{n}}, p_{n}\right)
$$

satisfy a system of inequalities to which Theorem 5.1 is applicable.

Let $S=\{\infty\} \cup\{p: p \mid b\}$ be the set of places on $\mathbb{Q}$ composed of the infinite place and the finite places corresponding to the prime divisors of $b$. We choose

$$
\Psi(\mathbf{x})=x_{1} \quad \text { for } \mathbf{x}=\left(x_{1}, x_{2}, x_{3}\right) \in \mathbb{Z}^{3} .
$$

We introduce the linear forms with real algebraic coefficients

$$
L_{1 \infty}(\mathbf{X})=X_{1}, \quad L_{2 \infty}(\mathbf{X})=X_{2}, \quad L_{3 \infty}(\mathbf{X})=-\xi X_{1}+\xi X_{2}+X_{3},
$$

and, for every prime divisor $p$ of $b$, we set

$$
L_{1 p}(\mathbf{X})=X_{1}, \quad L_{2 p}(\mathbf{X})=X_{2}, \quad L_{3 p}(\mathbf{X})=X_{3} .
$$

Set also

$$
e_{1 \infty}=1, \quad e_{2 \infty}=\frac{l+1}{k}, \quad e_{3 \infty}=-\varepsilon,
$$

and, for every prime divisor $p$ of $b$,

$$
e_{1 p}=\frac{\log |b|_{p}}{\log p}, \quad e_{2 p}=\frac{\log |b|_{p}}{\log p} \cdot \frac{l}{k}, \quad e_{3 p}=0 .
$$


Notice that

$$
\begin{aligned}
& \sum_{p \in S} \sum_{i=1}^{3} e_{i p}=-\left(\varepsilon-k^{-1}\right), \\
& e_{i \infty} \leq 1 \quad(i=1,2,3), \\
& e_{i p} \leq 0 \quad(p \in S \backslash\{\infty\}, i=1,2,3) .
\end{aligned}
$$

Furthermore,

$$
\operatorname{det}\left(L_{1 p}, L_{2 p}, L_{3 p}\right)=1 \quad \text { for } p \in S .
$$

Writing $d:=[\mathbb{Q}(\xi): \mathbb{Q}]$, we have

$$
\begin{aligned}
& \operatorname{Card}\left(\bigcup_{p \in S}\left\{L_{1 p}, L_{2 p}, L_{3 p}\right\}\right)=4, \\
& {\left[\mathbb{Q}\left(L_{i p}\right): \mathbb{Q}\right] \leq d \quad \text { for } p \in S, i=1,2,3 .}
\end{aligned}
$$

Further,

$$
\max _{p \in S, i=1,2,3} H^{*}\left(L_{i p}\right)=H(\xi) .
$$

(7.8)-(7.11) imply that the linear forms $L_{i p}$ and reals $e_{i p}$ defined above satisfy the conditions (5.1)-(5.6) of Theorem 5.1 with $n=3, R=4, D=d$, $H=H(\xi)$.

It is clear from (7.5), (7.8) that for any integer $n$ with (7.7) we have

$$
\left|L_{i p}\left(\mathbf{x}_{n}\right)\right|_{p} \leq \Psi\left(\mathbf{x}_{n}\right)^{e_{i p}} \quad \text { for } p \in S, i=1,2,3 .
$$

Assuming that $N$ is sufficiently large, we infer from (6.1), (7.4) that for every $n$ with (7.7) we have

$$
\Psi\left(\mathbf{x}_{n}\right)=b^{t_{n}} \geq 2^{2^{N / 2}-1}>\max \left\{2 H(\xi), 3^{6 /\left(\varepsilon-k^{-1}\right)}\right\} .
$$

Now, Theorem 5.1 implies that the set of vectors $\mathbf{x}_{n}=\left(b^{t_{n}}, b^{r_{n}}, p_{n}\right)$ with $n$ satisfying (7.7) is contained in the union of at most

$$
A_{1}:=8^{144}\left(1+\left(\varepsilon-k^{-1}\right)^{-1}\right)^{7} \log (8 d) \log \log (8 d)
$$

proper linear subspaces of $\mathbb{Q}^{3}$. We now consider the vectors $\mathbf{x}_{n}$ with $N / 2 \leq$ $n \leq N$ and drop the condition $l / k \leq r_{n} / t_{n}<(l+1) / k$. Then by (7.6), for any sufficiently large $N$, the set of vectors $\mathbf{x}_{n}=\left(b^{t_{n}}, b^{r_{n}}, p_{n}\right)$ with $N / 2 \leq n \leq N$ lies in the union of at most $k A_{1} \leq\left(\varepsilon^{-1}\right)^{8+\eta}$ proper linear subspaces of $\mathbb{Q}^{3}$.

We claim that if $N$ is sufficiently large, then any two-dimensional linear subspace of $\mathbb{Q}^{3}$ contains at most $\left(\varepsilon^{-1}\right)^{3+\eta}$ vectors $\mathbf{x}_{n}$. Having achieved this, it follows by (7.1), (7.4) that

$$
N / 2 \leq\left(\varepsilon^{-1}\right)^{8+\eta}\left(\varepsilon^{-1}\right)^{3+\eta} \leq N^{(11+2 \eta)(v+\eta)}=N^{1-\eta},
$$

which is clearly impossible if $N$ is sufficiently large. Thus (7.2) leads to a contradiction. 
So let $T$ be a two-dimensional linear subspace of $\mathbb{Q}^{3}$, say given by an equation $z_{1} X_{1}+z_{2} X_{2}+z_{3} X_{3}=0$, where we may assume that $z_{1}, z_{2}, z_{3}$ are integers without a common prime divisor. Let

$$
\mathcal{N}=\left\{i_{1}<\cdots<i_{r}\right\}
$$

be the set of $n$ with $N / 2 \leq n \leq N$ and $\mathbf{x}_{n} \in T$. So we have to prove that $r \leq\left(\varepsilon^{-1}\right)^{3+\eta}$.

Recall that by Lemma 6.1 , for every $n \geq 1$ we have either $r_{n}=0$, or $r_{n}>0$ and $b$ does not divide $p_{n}$. Hence the vectors $\mathbf{x}_{n}, n \geq 1$, are pairwise non-collinear. So the exterior product of $\mathbf{x}_{i_{1}}, \mathbf{x}_{i_{2}}$ must be a non-zero multiple of $\mathbf{z}=\left(z_{1}, z_{2}, z_{3}\right)$, and therefore

$$
\max \left\{\left|z_{1}\right|,\left|z_{2}\right|,\left|z_{3}\right|\right\} \leq 2 b^{2 t_{i_{2}}} .
$$

By combining (7.5) with $z_{1} b^{t_{n}}+z_{2} b^{r_{n}}+z_{3} p_{n}=0$, eliminating $b^{r_{n}}$, it follows that for $n$ in $\mathcal{N}$,

$$
\left|\frac{\xi\left(z_{1}+z_{2}\right)}{\xi z_{3}-z_{2}}-\frac{-p_{n}}{b^{t_{n}}}\right|<\left|\frac{z_{2}}{\xi z_{3}-z_{2}}\right|\left(b^{t_{n}}\right)^{-1-\varepsilon} .
$$

We want to apply Corollary 5.2 with $\xi\left(z_{1}+z_{2}\right) /\left(\xi z_{3}-z_{2}\right)$ instead of $\xi$.

Recall that $d$ denotes the degree of $\xi$. By (7.12), assuming that $N$ is sufficiently large, we have

$$
H\left(\frac{\xi\left(z_{1}+z_{2}\right)}{\xi z_{3}-z_{2}}\right) \leq 4 b^{2 t_{i_{2}}} H(\xi) \leq b^{3 t_{i_{2}}}
$$

Likewise,

$$
\left|\frac{z_{2}}{\xi z_{3}-z_{2}}\right| \leq H\left(\frac{\xi\left(z_{1}+z_{2}\right)}{\xi z_{3}-z_{2}}\right)^{d} \leq b^{3 d t_{i_{2}}} .
$$

There is no loss of generality to assume that there is an integer $k \leq r$ with

$$
b^{t_{i_{k}}} \geq b^{\left(3 d t_{i_{2}}\right)^{2 / \varepsilon}} .
$$

Indeed, if there is no such $k$ then we infer from (6.1) that

$$
b^{t_{i_{2}}^{r-3}} \leq b^{\left(3 d t_{i_{2}}\right)^{2 / \varepsilon}} \leq b^{t_{i_{2}}^{4 / \varepsilon}}
$$

hence

$$
r \leq 3+\frac{\log (4 / \varepsilon)}{\log 2},
$$

which is stronger than what we have to prove. Letting $k_{0}$ be the smallest integer $k$ with (7.14), we have

$$
b^{t_{i_{k_{0}}}} \geq b^{\left(3 d t_{i_{2}}\right)^{2 / \varepsilon}}, \quad k_{0} \leq 4+\frac{\log (4 / \varepsilon)}{\log 2} .
$$

Let $\mathcal{N}^{\prime}=\left\{i_{k_{0}}, i_{k_{0}+1}, \ldots, i_{r}\right\}$. We divide this set further into

$$
\mathcal{N}^{\prime \prime}=\left\{n \in \mathcal{N}^{\prime}: r_{n} \neq 0\right\}, \quad \mathcal{N}^{\prime \prime \prime}=\left\{n \in \mathcal{N}^{\prime}: r_{n}=0\right\} .
$$


By (7.13), for $n$ in $\mathcal{N}^{\prime \prime}$ we have

$$
\left|\frac{\xi\left(z_{1}+z_{2}\right)}{\xi z_{3}-z_{2}}-\frac{-p_{n}}{b^{t_{n}}}\right|<\left(b^{t_{n}}\right)^{-1-\varepsilon / 2} .
$$

Let $S_{1}=\emptyset$ and $S_{2}=\{p: p \mid b\}$. Then for $l \in S_{2}$ we have

$$
\left|b^{t_{n}}\right|_{l} \leq\left(b^{t_{n}}\right)^{\log |b|_{l} / \log b} .
$$

Lastly,

$$
b^{t_{n}} \geq b^{\left(3 d t_{i_{2}}\right)^{2 / \varepsilon}} \geq \max \left\{H\left(\frac{\xi\left(z_{1}+z_{2}\right)}{\xi z_{3}-z_{2}}\right), 2^{4 / \varepsilon}\right\} .
$$

Now, (7.16)-(7.18) imply that all the conditions of Corollary 5.2 are satisfied with $\varepsilon / 2$ instead of $\varepsilon$ and with

$$
x=p_{n}, \quad y=b^{t_{n}}, \quad f_{\infty}=1+\frac{\varepsilon}{2}, \quad f_{l}=-\frac{\log |b|_{l}}{\log b} \quad\left(l \in S_{2}\right) .
$$

Notice that

$$
f_{\infty}+\sum_{l \in S_{2}} f_{l}=2+\varepsilon / 2,
$$

and $f_{\infty} \geq 0, f_{l} \geq 0$ for $l \in S_{2}$. Consequently, the set of vectors $\left(p_{n}, b^{t_{n}}\right)$, $n \in \mathcal{N}^{\prime \prime}$, lies in the union of at most

$$
B(d, \varepsilon):=2^{32}\left(1+2 \varepsilon^{-1}\right)^{3} \log (6 d) \log \left(\left(1+2 \varepsilon^{-1}\right) \log (6 d)\right)
$$

one-dimensional linear subspaces of $\mathbb{Q}^{2}$. But the vectors $\left(p_{n}, b^{t_{n}}\right), n \in \mathcal{N}^{\prime \prime}$, are pairwise non-proportional, since $b$ does not divide $p_{n}$ for these values of $n$. Hence Card $\mathcal{N}^{\prime \prime} \leq B(d, \varepsilon)$.

To deal with $n \in \mathcal{N}^{\prime \prime \prime}$, we observe that by combining (7.5) again with $z_{1} b^{t_{n}}+z_{2} b^{r_{n}}+z_{3} p_{n}=0$, but now eliminating $p_{n}$, we obtain

$$
\left|\frac{\xi z_{3}+z_{1}}{\xi z_{3}-z_{2}}-\frac{1}{b^{t_{n}}}\right|<\left|\frac{z_{3}}{\xi z_{3}-z_{2}}\right|\left(b^{t_{n}}\right)^{-1-\varepsilon} .
$$

In precisely the same manner as above, one finds that the pairs $\left(b^{t_{n}}, 1\right)$ lie in at most $B(d, \varepsilon)$ one-dimensional subspaces. Since these pairs are pairwise non-proportional, it follows that Card $\mathcal{N}^{\prime \prime \prime} \leq B(d, \varepsilon)$.

By combining the above we obtain

$$
\operatorname{Card} \mathcal{N}=r \leq k_{0}+\operatorname{Card} \mathcal{N}^{\prime \prime}+\operatorname{Card} \mathcal{N}^{\prime \prime \prime} \leq k_{0}+2 B(d, \varepsilon) .
$$

In view of $(7.15),(7.19)$, this is smaller than $\left(\varepsilon^{-1}\right)^{3+\eta}$ for $N$ sufficiently large. This proves the claim, hence Theorem 2.1.

8. Proof of Theorem 3.1. We closely follow Section 4 of [8]. Assume without loss of generality that

$$
\frac{b-1}{b}<\xi<1 .
$$


Define the increasing sequence of positive integers $\left(n_{j}\right)_{j \geq 1}$ by $a_{1}=\cdots=a_{n_{1}}$, $a_{n_{1}} \neq a_{n_{1}+1}$ and $a_{n_{j}+1}=\cdots=a_{n_{j+1}}, a_{n_{j+1}} \neq a_{n_{j+1}+1}$ for $j \geq 1$. Observe that

$$
\operatorname{nbdc}(n, \xi, b)=\max \left\{j: n_{j} \leq n\right\}
$$

for $n \geq n_{1}$, and that $n_{j} \geq j$ for $j \geq 1$. Define

$$
\xi_{j}:=\sum_{k=1}^{n_{j}} \frac{a_{k}}{b^{k}}+\sum_{k=n_{j}+1}^{+\infty} \frac{a_{n_{j}+1}}{b^{k}}=\sum_{k=1}^{n_{j}} \frac{a_{k}}{b^{k}}+\frac{a_{n_{j}+1}}{b^{n_{j}}(b-1)} .
$$

Then

$$
\xi_{j}=\frac{P_{j}(b)}{b^{n_{j}}(b-1)},
$$

where $P_{j}(X)$ is an integer polynomial of degree at most $n_{j}$ whose constant coefficient $a_{n_{j}+1}-a_{n_{j}}$ is not divisible by $b$. That is, $b$ does not divide $P_{j}(b)$. We have

$$
\left|\xi-\xi_{j}\right|<\frac{1}{b^{n_{j+1}}}
$$

and this can be rewritten as

$$
\left|(b-1) \xi-\frac{P_{j}(b)}{b^{n_{j}}}\right|<\frac{b-1}{b^{n_{j+1}}} .
$$

By Liouville's inequality,

$$
\left|(b-1) \xi-\frac{P_{j}(b)}{b^{n_{j}}}\right| \geq\left(2 H((b-1) \xi) b^{n_{j}}\right)^{-d},
$$

so, if

$$
n_{j} \geq U:=1+3 H((b-1) \xi)
$$

then

$$
n_{j+1} \leq 2 d n_{j}
$$

In what follows, constants implied by the Vinogradov symbols $\ll, \gg$ are absolute. We need the following lemma.

Lemma 8.1. Let $0<\varepsilon \leq 1$ and let $j_{1}$ denote the smallest $j$ such that $n_{j} \geq \max \{U, 5 / \varepsilon\}$. Then

$$
\operatorname{Card}\left\{j: j \geq j_{1}, n_{j+1} / n_{j} \geq 1+2 \varepsilon\right\} \ll \log (6 d) \varepsilon^{-3} \log \left(\varepsilon^{-1} \log (6 d)\right) .
$$

Proof. For the integers $j$ under consideration, we have

$$
b^{n_{j}}>\max \left\{2 H((b-1) \xi), 2^{4 / \varepsilon}\right\} .
$$

Further, by (8.1), for $n_{j} \geq U$, we get

$$
\left|(b-1) \xi-\frac{P_{j}(b)}{b^{n_{j}}}\right|<\frac{b-1}{\left(b^{n_{j}}\right)^{1+2 \varepsilon}} \leq \frac{1}{\left(b^{n_{j}}\right)^{1+\varepsilon}} .
$$


Moreover, for every prime $l$ dividing $b$,

$$
\left|b^{n_{j}}\right|_{l} \leq\left(b^{n_{j}}\right)^{\log |b|_{l} / \log b} .
$$

Since

$$
1+\varepsilon+\sum_{l \mid b} \frac{-\log |b|_{l}}{\log b}=2+\varepsilon,
$$

Corollary 5.2 applied to (8.4), (8.5) shows that for the integers $j$ under consideration the pairs $\left(P_{j}(b), b^{n_{j}}\right)$ lie in

$$
\ll \log (6 d) \varepsilon^{-3} \log \left(\varepsilon^{-1} \log (6 d)\right)
$$

one-dimensional linear subspaces of $\mathbb{Q}^{2}$. But these pairs are non-proportional since $b$ does not divide $P_{j}(b)$. The lemma follows.

Let $j_{0}$ be the smallest $j$ such that $n_{j} \geq U$. Let $J$ be an integer with

$$
J>\max \left\{n_{j_{0}}^{3},(4 d)^{6}\right\} .
$$

Let $j_{2}$ be the largest integer with

$$
n_{j_{2}} \leq 6 d J^{1 / 3} .
$$

Then since $n_{j_{2}} \geq n_{j_{0}} \geq U$, we have

$$
n_{j_{2}} \geq \frac{n_{j_{2}+1}}{2 d} \geq 3 J^{1 / 3} .
$$

Now choose

$$
\varepsilon_{1}:=\left(\frac{\log (6 d) \log J}{J}\right)^{1 / 3}
$$

and let $k$ be any positive integer and $\varepsilon_{2}, \ldots, \varepsilon_{k-1}$ any reals such that

$$
\varepsilon_{1}<\varepsilon_{2}<\cdots<\varepsilon_{k-1}<\varepsilon_{k}:=1 \text {. }
$$

We infer from (8.8) that

$$
n_{j_{2}} \geq \max \left\{U, 5 / \varepsilon_{h}\right\} \quad \text { for } h=1, \ldots, k \text {. }
$$

Let $\mathcal{S}_{0}=\left\{j_{2}, j_{2}+1, \ldots, J\right\}$ and, for $h=1, \ldots, k$, let $\mathcal{S}_{h}$ denote the set of positive integers $j$ such that $j_{2} \leq j \leq J$ and $n_{j+1} \geq\left(1+2 \varepsilon_{h}\right) n_{j}$. Further, let $T_{h}$ be the cardinality of $\mathcal{S}_{h}$ for $h=1, \ldots, k$. Obviously, $\mathcal{S}_{0} \supset \mathcal{S}_{1} \supset \cdots \supset \mathcal{S}_{k}$ and

$$
\mathcal{S}_{0}=\left(\mathcal{S}_{0} \backslash \mathcal{S}_{1}\right) \cup\left(\mathcal{S}_{1} \backslash \mathcal{S}_{2}\right) \cup \cdots \cup\left(\mathcal{S}_{k-1} \backslash \mathcal{S}_{k}\right) \cup \mathcal{S}_{k} .
$$

Now,

$$
\begin{aligned}
\frac{n_{J}}{n_{j_{2}}}=\frac{n_{J}}{n_{J-1}} \cdot \frac{n_{J-1}}{n_{J-2}} \cdots \frac{n_{j_{2}+1}}{n_{j_{2}}} & =\prod_{h=0}^{k-1}\left(\prod_{j \in \mathcal{S}_{h} \backslash \mathcal{S}_{h+1}} \frac{n_{j+1}}{n_{j}}\right)\left(\prod_{j \in \mathcal{S}_{h}} \frac{n_{j+1}}{n_{j}}\right) \\
& \leq\left(1+2 \varepsilon_{1}\right)^{J} \prod_{h=1}^{k-1}\left(1+2 \varepsilon_{h+1}\right)^{T_{h}-T_{h+1}(2 d)^{T_{k}}}
\end{aligned}
$$


where in the last estimate we have used (8.11) and (8.3). Taking logarithms, we get

$$
\begin{aligned}
\log \left(n_{J} / n_{j_{2}}\right) & \leq 2 \varepsilon_{1} J+2 \sum_{h=1}^{k-1} \varepsilon_{h+1}\left(T_{h}-T_{h+1}\right)+T_{k} \log (2 d) \\
& \leq 2 \varepsilon_{1} J+2 \varepsilon_{2} T_{1}+2 \sum_{h=2}^{k-1}\left(\varepsilon_{h+1}-\varepsilon_{h}\right) T_{h}-2 T_{k}+T_{k} \log (2 d) .
\end{aligned}
$$

In view of (8.11), we can apply Lemma 8.1 to obtain

$$
T_{h} \ll \log (6 d) \varepsilon_{h}^{-3} \log \left(\varepsilon_{h}^{-1} \log (6 d)\right)
$$

for $h=1, \ldots, k$. This gives

$$
\begin{aligned}
\log \left(n_{J} / n_{j_{2}}\right) \ll & \varepsilon_{1} J+\log (6 d) \varepsilon_{2} \varepsilon_{1}^{-3} \log \left(\varepsilon_{1}^{-1} \log (6 d)\right) \\
& +\log (6 d) \sum_{h=2}^{k-1} \varepsilon_{h}^{-3} \log \left(\varepsilon_{h}^{-1} \log (6 d)\right)\left(\varepsilon_{h+1}-\varepsilon_{h}\right) \\
& +(\log (6 d))^{2} \log \log (6 d) .
\end{aligned}
$$

Now, let $k$ tend to infinity and $\max _{1 \leq h \leq k-1}\left(\varepsilon_{h+1}-\varepsilon_{h}\right)$ tend to zero. Then the sum converges to a Riemann integral, and, after a short computation, using the fact that in view of $(8.6),(8.9)$ we have $\varepsilon_{1}^{-1} \gg d$, we get

$$
\log \left(n_{J} / n_{j_{2}}\right) \ll \varepsilon_{1} J+\log (6 d) \varepsilon_{1}^{-2} \log \left(\varepsilon_{1}^{-1}\right) .
$$

By (8.6) and (8.7), we have $n_{j_{2}} \leq J^{1 / 2} \leq n_{J}^{1 / 2}$, so $n_{J} / n_{j_{2}} \geq n_{J}^{1 / 2}$. Inserting our choice (8.9) for $\varepsilon_{1}$ and using (8.6), we get

$$
\log n_{J} \ll J^{2 / 3}(\log J)^{1 / 3}(\log (6 d))^{1 / 3},
$$

i.e.,

$$
J \gg\left(\log n_{J}\right)^{3 / 2}\left(\log \log n_{J}\right)^{-1 / 2}(\log (6 d))^{-1 / 2} .
$$

This proves Theorem 3.1 .

9. Final remarks. We deduce from Corollary 5.2 an improvement of an extension due to Mahler [17] of a theorem of Cugiani [11] (see [9] for further references on the Cugiani-Mahler theorem).

Let $S_{1}, S_{2}$ be finite, possibly empty sets of prime numbers, put $S:=$ $\{\infty\} \cup S_{1} \cup S_{2}$, let $\xi \in \overline{\mathbb{Q}}$ be an algebraic number, let $\varepsilon>0$, and let $f_{p}$ $(p \in S)$ be reals such that

$$
f_{p} \geq 0 \quad \text { for } p \in S, \quad \sum_{p \in S} f_{p}=2 .
$$

Let $\varepsilon: \mathbb{Z}_{\geq 1} \rightarrow \mathbb{R}_{>0}$ be a non-increasing function. We consider the system of inequalities 


$$
\left\{\begin{array}{ll}
|\xi-x / y| \leq y^{-f_{\infty}-\varepsilon(y)} \\
|x|_{p} \leq y^{-f_{p}} \quad\left(p \in S_{1}\right) \\
|y|_{p} \leq y^{-f_{p}} \quad\left(p \in S_{2}\right)
\end{array}\right\} \quad \begin{aligned}
& \text { in }(x, y) \in \mathbb{Z}^{2} \text { with } y>0 \\
& \text { and } \operatorname{gcd}(x, y)=1 .
\end{aligned}
$$

Arguing as in [9], we get the following improvement of Theorem 1 on p. 169 of [17], which we state without proof. For a positive integer $m$, we denote by $\exp _{m}$ the $m$ th iterate of the exponential function and by $\log _{m}$ the function that coincides with the $m$ th iterate of the logarithm function on $\left[\exp _{m} 1,+\infty\right)$ and that takes the value 1 on $\left(-\infty, \exp _{m} 1\right]$.

TheOrem 9.1. Keep the above notation. Let $m$ be a positive integer, and $c$ be a positive real number. Set

$$
\varepsilon(y)=c\left(\log _{m+1} y\right)^{-1 / 3} \log _{m+2} y \quad \text { for } y \geq 1 .
$$

Let $\left(x_{j} / y_{j}\right)_{j \geq 1}$ be the sequence of reduced rational solutions of (9.1) ordered so that $1 \leq y_{1}<y_{2}<\cdots$. Then either the sequence $\left(x_{j} / y_{j}\right)_{j \geq 1}$ is finite or

$$
\limsup _{j \rightarrow+\infty} \frac{\log _{m} y_{j+1}}{\log _{m} y_{j}}=+\infty .
$$

Theorem 9.1 improves upon Mahler's result, which deals only with the case $m=1$ and involves the very slowly decreasing function $y \mapsto\left(\log _{3} y\right)^{-1 / 2}$.

Theorem 9.1 can be compared with Theorem 2 from [9] that deals with products of linear forms and involves a function $\varepsilon$ that depends on the cardinality of $S_{1} \cup S_{2}$. Note that Theorem 6.5.10 from Chapter 6 of the monograph of Bombieri and Gubler [6], given without proof, deals also with products of linear forms, but the function $\varepsilon$ occurring there does not involve the cardinality of $S_{1} \cup S_{2}$.

We can then proceed exactly as Mahler did ([17, Theorem 3, p. 178]) to construct new explicit examples of transcendental numbers.

THeOREM 9.2. Let $b \geq 2$ be an integer. Let $\theta$ be a real number with $0<\theta<1$. Let $\mathbf{n}=\left(n_{j}\right)_{j \geq 1}$ be an increasing sequence of positive integers satisfying $n_{1} \geq 3$ and

$$
n_{j+1} \geq\left(1+\frac{\log \log n_{j}}{\left(\log n_{j}\right)^{1 / 3}}\right) n_{j}, \quad j \geq 1 .
$$

Let $\left(a_{j}\right)_{j \geq 1}$ be a sequence of positive integers prime to $b$ such that

$$
a_{j+1} \leq b^{\theta\left(n_{j+1}-n_{j}\right)}, \quad j \geq 1 .
$$

Then the real number

$$
\xi=\sum_{j \geq 1} a_{j} b^{-n_{j}}
$$

is transcendental. 
This improves Theorem 4 from [8], which is, in addition, incorrectly formulated.

We omit the proof of Theorem 9.2, which follows from Theorem 9.1 with $m=1$.

It is of interest to note that Theorem 9.2 yields Corollary 3.2 only for $\eta>3 / 4$. We would have obtained the same result by taking $k=1$ in (8.10). It is precisely the introduction of the parameter $k$ there that allows us to get in Theorem 3.1 the exponent of $\log n$ equal to $3 / 2$ and not to $4 / 3$.

\section{Appendix. A quantitative two-dimensional parametric subspace} theorem. We give a proof of the two-dimensional case of Proposition 4.1. We keep the notation and assumptions from Section 4, except that we assume $n=2$. As before, $\mathbb{K}$ is an algebraic number field. We recall the notation from Section 4 , but now specialized to $n=2$. Thus, $\mathcal{L}=\left(L_{i v}: v \in M_{\mathbb{K}}, i=\right.$ $1,2)$ is a tuple of linear forms satisfying

(A.1) $\quad L_{i v} \in \mathbb{K}\left[X_{1}, X_{2}\right] \quad$ for $v \in M_{\mathbb{K}}, i=1,2$,

(A.2) $L_{1 v}=X_{1}, L_{2 v}=X_{2} \quad$ for all but finitely many $v \in M_{\mathbb{K}}$,

(A.3) $\quad \operatorname{det}\left(L_{1 v}, L_{2 v}\right)=1 \quad$ for $v \in M_{\mathbb{K}}$,$$
\operatorname{Card}\left(\bigcup_{v \in M_{\mathbb{K}}}\left\{L_{1 v}, L_{2 v}\right\}\right) \leq r,
$$

and $\mathbf{c}=\left(c_{i v}: v \in M_{\mathbb{K}}, i=1,2\right)$ is a tuple of reals satisfying

$$
c_{1 v}=c_{2 v}=0 \quad \text { for all but finitely many } v \in M_{\mathbb{K}},
$$

$$
\sum_{v \in M_{\mathbb{K}}} \sum_{i=1}^{2} c_{i v}=0 \text {, }
$$

$$
\sum_{v \in M_{\mathbb{K}}} \max \left(c_{1 v}, c_{2 v}\right) \leq 1
$$

We define

$$
\mathcal{H}=\mathcal{H}(\mathcal{L}):=\prod_{v \in M_{\mathbb{K}}} \max _{1 \leq i_{1}<i_{2} \leq s}\left\|\operatorname{det}\left(L_{i_{1}}, L_{i_{2}}\right)\right\|_{v}
$$

where we have written $\left\{L_{1}, \ldots, L_{s}\right\}$ for $\bigcup_{v \in M_{\mathbb{K}}}\left\{L_{1 v}, L_{2 v}\right\}$. Finally, for any finite extension $\mathbb{E}$ of $\mathbb{K}$ and any place $w \in M_{\mathbb{E}}$ we define

$$
L_{i w}=L_{i v}, \quad c_{i w}=d(w \mid v) c_{i v} \quad \text { for } i=1,2,
$$

where $v$ is the place of $M_{\mathbb{K}}$ lying below $w$ and $d(w \mid v)$ is given by (4.2).

The twisted height $H_{Q, \mathcal{L}, \mathbf{c}}(\mathbf{x})$ of $\mathbf{x} \in \overline{\mathbb{Q}}^{2}$ is defined by taking any finite extension $\mathbb{E}$ of $\mathbb{K}$ such that $\mathbf{x} \in \mathbb{E}^{2}$ and putting 


$$
H_{Q, \mathcal{L}, \mathbf{c}}(\mathbf{x}):=\prod_{w \in M_{\mathbb{E}}} \max _{i=1,2}\left\|L_{i w}(\mathbf{x})\right\|_{w} Q^{-c_{i w}} ;
$$

this does not depend on the choice of $\mathbb{E}$.

Proposition A.1. Let $\mathcal{L}=\left(L_{i v}: v \in M_{\mathbb{K}}, i=1,2\right)$ be a tuple of linear forms and $\mathbf{c}=\left(c_{i v}: v \in M_{\mathbb{K}}, i=1,2\right)$ a tuple of reals satisfying (A.1)-(A.7). Further, let $0<\delta \leq 1$. Then there are one-dimensional linear subspaces $T_{1}, \ldots, T_{t_{2}}$ of $\overline{\mathbb{Q}}^{2}$, all defined over $\mathbb{K}$, with

$$
t_{2}=t_{2}(r, \delta)=2^{25} \delta^{-3} \log (2 r) \log \left(\delta^{-1} \log (2 r)\right)
$$

such that for every real $Q$ with

$$
Q>\max \left(\mathcal{H}^{2 / r(r-1)}, 4^{1 / \delta}\right)
$$

there is a subspace $T_{i} \in\left\{T_{1}, \ldots, T_{t_{2}}\right\}$ with

$$
\left\{\mathbf{x} \in \overline{\mathbb{Q}}^{2}: H_{Q, \mathcal{L}, \mathbf{c}}(\mathbf{x}) \leq Q^{-\delta}\right\} \subset T_{i} .
$$

The proof of Proposition A.1 is by combining some lemmata from [14], specialized to $n=2$. We keep the notation and assumptions from above. By condition (A.4), there exists a "family" (unordered sequence, possibly with repetitions) of linear forms $\left\{L_{1}, \ldots, L_{r}\right\}$ such that $L_{1 v}, L_{2 v}$ belong to this family for every $v \in M_{\mathbb{K}}$ and $L_{1}=X_{1}, L_{2}=X_{2}$. Now conditions (A.1)-(A.7) imply the conditions (5.12)-(5.17) on p. 36 of [14] with $n=2$. These conditions are kept throughout [14] and so all arguments of [14] from p. 36 onwards are applicable in our situation. Since in what follows the tuples $\mathcal{L}$ and $\mathbf{c}$ will be fixed and only $Q$ will vary, we will write $H_{Q}$ for the twisted height $H_{Q, \mathcal{L}, \mathbf{c}}$.

Let $Q$ be a real with $Q \geq 1$. We define the "successive infima" $\lambda_{1}(Q)$, $\lambda_{2}(Q)$ of $H_{Q}$ as follows: for $i=1,2, \lambda_{i}(Q)$ is the infimum of all reals $\lambda>0$ such that $\left\{\mathbf{x} \in \overline{\mathbb{Q}}^{2}: H_{Q}(\mathbf{x}) \leq \lambda\right\}$ contains at least $i$ linearly independent points. Since we are working on the algebraic closure of $\mathbb{Q}$ and not on a given number field, those infima need not be assumed by $H_{Q}$.

In [14] (specialized to $n=2$ ), $\lambda_{1}(Q), \lambda_{2}(Q)$ were defined to be the successive infima of some sort of parallelepiped $\Pi(Q, \mathbf{c})$ defined over $\overline{\mathbb{Q}}$, and the lemmata in that paper were all formulated in terms of those infima. However, according to [14, Corollary 7.4, p. 53], applied with $n=2$ and $\mathbf{A}=\left(Q^{c_{i v}}, v \in M_{\mathbb{K}}, i=1,2\right)$, the successive infima of $\Pi(Q, \mathbf{c})$ are equal to the successive infima of $H_{Q}$ as defined above.

Lemma A.2. Let $\delta>0, Q \geq 1$.

(i) $1 / 2 \leq \lambda_{1}(Q) \lambda_{2}(Q) \leq 2$.

(ii) If there exists a non-zero $\mathbf{x} \in \overline{\mathbb{Q}}^{2}$ with $H_{Q}(\mathbf{x}) \leq Q^{-\delta}$ then $\lambda_{1}(Q) \leq$ $Q^{-\delta}$ and $\lambda_{2}(Q) \geq \frac{1}{2} Q^{\delta}$. 
Proof. Assertion (i) follows from [14, Corollary 7.6, p. 54]; (ii) is then obvious.

Lemma A.3 (Gap Principle). Let $\delta>0$, and let $Q_{0}$ be a real with $Q_{0}>$ $4^{1 / \delta}$. Then there is a unique, one-dimensional linear subspace $T$ of $\overline{\mathbb{Q}}^{2}$ with the following property: for every $Q$ with

$$
Q_{0} \leq Q<Q_{0}^{1+\delta / 2}
$$

we have $\left\{\mathbf{x} \in \overline{\mathbb{Q}}^{2}: H_{Q}(\mathbf{x}) \leq Q^{-\delta}\right\} \subset T$.

Proof. Let $T$ be the linear subspace of $\overline{\mathbb{Q}}^{2}$ spanned by all $\mathbf{x}$ such that $H_{Q_{0}}(\mathbf{x}) \leq Q_{0}^{-\delta / 2}$. If $T \neq(\mathbf{0})$ then by Lemma A.2 we have $\lambda_{1}\left(Q_{0}\right) \leq Q_{0}^{-\delta / 2}$ and $\lambda_{2}\left(Q_{0}\right) \geq \frac{1}{2} Q_{0}^{\delta / 2}$, which by our assumption on $Q_{0}$ is strictly larger than $\lambda_{1}\left(Q_{0}\right)$. Hence $T$ has dimension at most 1 . So it suffices to prove that if $\mathbf{x} \in \overline{\mathbb{Q}}^{2}$ and $Q$ are such that $Q_{0} \leq Q<Q_{0}^{1+\delta / 2}$ and $H_{Q}(\mathbf{x}) \leq Q^{-\delta}$, then $H_{Q_{0}}(\mathbf{x}) \leq Q_{0}^{-\delta / 2}$.

To prove this, choose a finite extension $\mathbb{E}$ of $\mathbb{K}$ such that $\mathbf{x} \in \mathbb{E}^{2}$. Notice that by (A.7), (A.9), (4.3) we have $u:=\sum_{w \in M_{\mathbb{E}}} \max \left(c_{1 w}, c_{2 w}\right) \leq 1$. For $w \in M_{\mathbb{E}}$ we have

$$
\begin{aligned}
\max \left(\frac{\left\|L_{1 w}(\mathbf{x})\right\|_{w}}{Q_{0}^{c_{1 w}}}, \frac{\left\|L_{2 w}(\mathbf{x})\right\|_{w}}{Q_{0}^{c_{2 w}}}\right) & \\
\leq & \max \left(\frac{\left\|L_{1 w}(\mathbf{x})\right\|_{w}}{Q^{c_{1 w}}}, \frac{\left\|L_{2 w}(\mathbf{x})\right\|_{w}}{Q^{c_{2 w}}}\right) \cdot\left(\frac{Q}{Q_{0}}\right)^{\max \left(c_{1 w}, c_{2 w}\right)} .
\end{aligned}
$$

So

$$
H_{Q_{0}}(\mathbf{x}) \leq H_{Q}(\mathbf{x})\left(\frac{Q}{Q_{0}}\right)^{u} \leq Q^{-\delta} \cdot \frac{Q}{Q_{0}} \leq Q_{0}^{-\delta} Q_{0}^{\delta / 2}=Q_{0}^{-\delta / 2} .
$$

Lemma A.4. Let $\delta>0$ and let $A, B$ be reals with $4^{1 / \delta}<A<B$. Then there are one-dimensional linear subspaces $T_{1}, \ldots, T_{t_{3}}$ of $\overline{\mathbb{Q}}^{2}$ with

$$
t_{3} \leq 1+\frac{\log (\log B / \log A)}{\log (1+\delta / 2)}
$$

such that for every $Q$ with $A \leq Q<B$ there is $T_{i} \in\left\{T_{1}, \ldots, T_{t_{3}}\right\}$ with

$$
\left\{\mathbf{x} \in \overline{\mathbb{Q}}^{2}: H_{Q}(\mathbf{x}) \leq Q^{-\delta}\right\} \subset T_{i} .
$$

Proof. Let $k$ be the smallest integer with $A^{(1+\delta / 2)^{k}} \geq B$. Apply Lemma A.3 with $Q_{0}=A^{(1+\delta / 2)^{i}}$ for $i=0, \ldots, k-1$.

We define the Euclidean height $H_{2}(\mathbf{x})$ for $\mathbf{x}=\left(x_{1}, \ldots, x_{m}\right) \in \overline{\mathbb{Q}}^{m}$ as follows. Choose any number field $\mathbb{E}$ such that $\mathbf{x} \in \mathbb{E}^{m}$, define

$$
\|\mathbf{x}\|_{w, 2}:= \begin{cases}\left\{\left(\sum_{i=1}^{m}\left|x_{i}\right|_{w}^{2}\right)^{1 / 2}\right\}^{\left[\mathbb{E}_{w}: \mathbb{R}\right] /[\mathbb{E}: \mathbb{Q}]} & \text { if } w \text { is Archimedean, } \\ \max \left(\left\|x_{1}\right\|_{w}, \ldots,\left\|x_{m}\right\|_{w}\right) & \text { if } w \text { is non-Archimedean }\end{cases}
$$


and put

$$
H_{2}(\mathbf{x}):=\prod_{w \in M_{\mathbb{E}}}\|\mathbf{x}\|_{w, 2}
$$

This is independent of the choice of $\mathbb{E}$. For a polynomial $P$ with coefficients in $\overline{\mathbb{Q}}$, define $H_{2}(P):=H_{2}(\mathbf{p})$, where $\mathbf{p}$ is a vector consisting of the coefficients of $P$.

Lemma A.5. Let $\delta>0$. Consider the set of reals $Q$ such that

$$
\begin{aligned}
& \text { there is } \mathbf{x}_{Q} \in \overline{\mathbb{Q}}^{2} \backslash\{\mathbf{0}\} \text { with } H_{Q}\left(\mathbf{x}_{Q}\right) \leq Q^{-\delta}, \\
& Q^{\delta}>(2 \mathcal{H})^{6\left(\begin{array}{c}
r \\
2
\end{array}\right) .}
\end{aligned}
$$

Then one of the following two alternatives is true:

(i) For all $Q$ under consideration we have $H_{2}\left(\mathbf{x}_{Q}\right)>Q^{\delta / 3\left(\begin{array}{l}r \\ 2\end{array}\right)}$.

(ii) There is a single one-dimensional linear subspace $T_{0}$ of $\overline{\mathbb{Q}}^{2}$ such that for all $Q$ under consideration we have $\mathbf{x}_{Q} \in T_{0}$.

Proof. This is $[14$, p. 80 , Lemma 12.4] with $n=2$. Condition (A.14) and Lemma A.2 imply $\lambda_{1}(Q) \leq Q^{-\delta}$, which is condition (12.37) of Lemma 12.4 of [14] with $n=2$. Further, the quantity $R$ in that lemma is $\leq\left(\begin{array}{l}r \\ 2\end{array}\right)$ (see [14, p. 75 , Lemma 12.1]).

Let $m$ be a positive integer and $\mathbf{r}=\left(r_{1}, \ldots, r_{m}\right)$ a tuple of positive integers. We say that a polynomial is multihomogeneous of degree $\mathbf{r}$ in the blocks of variables $\mathbf{X}_{1}=\left(X_{11}, X_{12}\right), \ldots, \mathbf{X}_{m}=\left(X_{m 1}, X_{m 2}\right)$ if it can be expressed as a linear combination of monomials

$$
\prod_{h=1}^{m} \prod_{k=1}^{2} X_{h k}^{i_{h k}} \quad \text { with } i_{h 1}+i_{h 2}=r_{h} \text { for } h=1, \ldots, m .
$$

(Below, $h$ will always index the block.) Given points $\mathbf{x}_{h}=\left(x_{h 1}, x_{h 2}\right)(h=$ $1, \ldots, m)$ and a polynomial $P$ which is multihomogeneous in $\mathbf{X}_{1}, \ldots, \mathbf{X}_{m}$, we write $P\left(\mathbf{x}_{1}, \ldots, \mathbf{x}_{m}\right)$ for the value obtained by substituting $x_{h k}$ for $X_{h k}$ $(h=1, \ldots, m, k=1,2)$.

The index of a polynomial $P$ multihomogeneous in $\mathbf{X}_{1}, \ldots, \mathbf{X}_{m}$ with respect to points $\mathbf{x}_{1}, \ldots, \mathbf{x}_{m}$ and to a tuple of positive integers $\mathbf{r}=\left(r_{1}, \ldots, r_{m}\right)$, denoted by

$$
\operatorname{Ind}\left(P ; \mathbf{r} ; \mathbf{x}_{1}, \ldots, \mathbf{x}_{m}\right),
$$

is defined to be the smallest real $\sigma$ with the following property: there is a tuple of non-negative integers $\mathbf{i}=\left(i_{h k}: h=1, \ldots, m, k=1,2\right)$ such that

$$
\left(\prod_{h=1}^{m} \prod_{k=1}^{2}\left(\frac{\partial}{\partial X_{h k}}\right)^{i_{h k}} P\right)\left(\mathbf{x}_{1}, \ldots, \mathbf{x}_{m}\right) \neq 0, \quad \sum_{h=1}^{m} \frac{i_{h 1}+i_{h 2}}{r_{h}}=\sigma .
$$


For a field $\mathbb{F}$ and a tuple $\mathbf{r}=\left(r_{1}, \ldots, r_{m}\right)$ of positive integers, we denote by $\mathbb{F}[\mathbf{r}]$ the set of polynomials with coefficients in $\mathbb{F}$ which are multihomogeneous of degree $\mathbf{r}$ in $\mathbf{X}_{1}, \ldots, \mathbf{X}_{m}$.

We define the constant $C(\mathbb{K}):=\left|D_{\mathbb{K}}\right|^{1 /[\mathbb{K}: \mathbb{Q}]}$, where $D_{\mathbb{K}}$ denotes the discriminant of $\mathbb{K}$. In fact, the precise value of $C(\mathbb{K})$ is not of importance.

Lemma A.6. Suppose that $0<\delta \leq 1$, let $\theta$ be a real with

$$
0<\theta \leq \delta / 80
$$

$m$ an integer with

$$
m>4 \theta^{-2} \log (2 r)
$$

and $\mathbf{r}=\left(r_{1}, \ldots, r_{m}\right)$ a tuple of positive integers, and put $q:=r_{1}+\cdots+r_{m}$. Suppose that there exist positive reals $Q_{1}, \ldots, Q_{m}$ and non-zero points $\mathbf{x}_{1}, \ldots, \mathbf{x}_{m}$ in $\overline{\mathbb{Q}}^{2}$ such that

(A.18) $r_{1} \log Q_{1} \leq r_{h} \log Q_{h} \leq(1+\theta) r_{1} \log Q_{1} \quad(h=1, \ldots, m)$,

(A.19) $\quad H_{Q_{h}}\left(\mathbf{x}_{h}\right) \leq Q_{h}^{-\delta} \quad(h=1, \ldots, m)$,

(A.20) $\quad Q_{h}^{\delta}>C(\mathbb{K})^{5 / 4 q} 2^{50} \mathcal{H}^{5} \theta^{-5 / 2}$.

Then there is a non-zero polynomial $P \in K[\mathbf{r}]$ such that

$$
\begin{aligned}
& \operatorname{Ind}\left(P ; \mathbf{r} ; \mathbf{x}_{1}, \ldots, \mathbf{x}_{m}\right) \geq m \theta, \\
& H_{2}(P) \leq C(\mathbb{K})^{1 / 2} 2^{3 m}(12 \mathcal{H})^{q} .
\end{aligned}
$$

Proof. This is [14, Lemma 15.1 , p. 89] with $n=2$. The space $V_{[h]}\left(Q_{h}\right)$ in that lemma is in our situation precisely the space spanned by $\mathbf{x}_{h}$ for $h=1, \ldots, m$. Inequality (A.17) comes from [14, (14.7), p. 83]; later it is assumed that $s=r$ (see [14, (14.10), p. 85]). Inequality (A.22) comes from the inequality at the bottom of p. 87 of [14]. The construction of the polynomial $P$ is by means of a now standard argument, based on the Bombieri-Vaaler Siegel's lemma.

Lemma A.7 (Roth's lemma). Let $0<\theta \leq 1$. Let $m$ be an integer with $m \geq 2$ and $\mathbf{r}=\left(r_{1}, \ldots, r_{m}\right)$ a tuple of positive integers such that

$$
\frac{r_{h}}{r_{h+1}} \geq \frac{2 m^{2}}{\theta} \quad(h=1, \ldots, m-1) .
$$

Further, let $P$ be a non-zero polynomial in $\overline{\mathbb{Q}}[\mathbf{r}]$ and $\mathbf{x}_{1}, \ldots, \mathbf{x}_{m}$ non-zero points in $\overline{\mathbb{Q}}^{2}$ such that

$$
H_{2}\left(\mathbf{x}_{h}\right)^{r_{h}} \geq\left(e^{q} H_{2}(P)\right)^{\left(3 m^{2} / \theta\right)^{m}} \quad(h=1, \ldots, m),
$$

where $e=2.7182 \ldots, q=r_{1}+\cdots+r_{m}$. Then

$$
\operatorname{Ind}\left(P ; \mathbf{r} ; \mathbf{x}_{1}, \ldots, \mathbf{x}_{m}\right)<m \theta .
$$

Proof. This is the case $n=2$ of [13, Lemma 24]. It is an immediate consequence of [12, Theorem 3]. 
We keep our assumption $0<\delta \leq 1$ and define the integer

$$
m:=1+\left[25600 \delta^{-2} \log (2 r)\right] .
$$

Put

$$
C:=(36 \mathcal{H})^{m\left(240 m^{2} / \delta\right)^{m} 3\left(\begin{array}{l}
r \\
2
\end{array}\right) / \delta} .
$$

Denote by $\mathcal{S}$ the set of reals $Q$ such that

$$
Q \geq C, \quad \text { there is } \mathbf{x} \in \overline{\mathbb{Q}}^{2} \backslash\{\mathbf{0}\} \text { with } H_{Q}(\mathbf{x}) \leq Q^{-\delta} .
$$

Lemma A.8. One of the following two alternatives is true:

(i) There is a single, one-dimensional linear subspace $T_{0}$ of $\overline{\mathbb{Q}}^{2}$ such that for every $Q \in \mathcal{S}$ we have $\left\{\mathbf{x} \in \overline{\mathbb{Q}}^{2}: H_{Q}(\mathbf{x}) \leq Q^{-\delta}\right\} \subset T_{0}$.

(ii) There are reals $Q_{1}, \ldots, Q_{m-1}$ with $C \leq Q_{1}<\cdots<Q_{m-1}$ such that

$$
\mathcal{S} \subset \bigcup_{h=1}^{m-1}\left[Q_{h}, Q_{h}^{162 m^{2} / \delta}\right] .
$$

Proof. Suppose that neither (i) nor (ii) is true. We will deduce that there are a tuple $\mathbf{r}=\left(r_{1}, \ldots, r_{m}\right)$ of positive integers, a non-zero polynomial $P \in \mathbb{K}[\mathbf{r}]$, and non-zero points $\mathbf{x}_{1}, \ldots, \mathbf{x}_{m} \in \overline{\mathbb{Q}}^{2}$, satisfying both (A.21) and (A.25). This is obviously impossible.

By our assumption, there are reals $Q_{1}, \ldots, Q_{m} \in \mathcal{S}$ with

$$
\frac{\log Q_{h+1}}{\log Q_{h}} \geq \frac{162 m^{2}}{\delta} \quad(h=1, \ldots, m-1),
$$

and non-zero points $\mathbf{x}_{1}, \ldots, \mathbf{x}_{m} \in \overline{\mathbb{Q}}^{2}$ with

$$
H_{Q_{h}}\left(\mathbf{x}_{h}\right) \leq Q_{h}^{-\delta} \quad(h=1, \ldots, m) .
$$

Put $\theta:=\delta / 80$. First choose a positive integer $s_{1}$ such that $\theta s_{1} \log Q_{1}>$ $\log Q_{h}$ for $h=2, \ldots, m$. Then there are integers $s_{2}, \ldots, s_{m}$ such that

$$
s_{1} \log Q_{1} \leq s_{h} \log Q_{h} \leq(1+\theta) s_{1} \log Q_{1} \quad(h=1, \ldots, m) .
$$

Now take $r_{h}:=t s_{h}(h=1, \ldots, m), \mathbf{r}=\left(r_{1}, \ldots, r_{m}\right)$, where $t$ is a positive integer, chosen large enough so that the right-hand side of (A.20) is smaller than $C^{\delta}$ and the right-hand side of $(\mathrm{A} .22)$ is smaller than $(13 \mathcal{H})^{q}$, where $q=r_{1}+\cdots+r_{m}$. Then conditions (A.18)-(A.20) of Lemma A.6 are satisfied, hence there exists a non-zero polynomial $P \in \mathbb{K}[\mathbf{r}]$ such that (A.21), (A.22) are satisfied. So we have in fact

$$
H_{2}(P) \leq(13 \mathcal{H})^{q} .
$$

We now show that $P, \mathbf{r}, \mathbf{x}_{1}, \ldots, \mathbf{x}_{m}$ satisfy conditions (A.23), (A.24) of Lemma A.7. Then it follows that (A.25) holds, and we arrive at the contradiction we wanted. 
In view of $(\mathrm{A} .30),(\mathrm{A} .18)$ and $\theta=\delta / 80 \leq 1 / 80$ we have

$$
\frac{r_{h}}{r_{h+1}}=\frac{r_{h} \log Q_{h}}{r_{h+1} \log Q_{h+1}} \cdot \frac{\log Q_{h+1}}{\log Q_{h}} \geq \frac{1}{1+\theta} \cdot \frac{162 m^{2}}{\delta} \geq \frac{160 m^{2}}{\delta}=\frac{2 m^{2}}{\theta}
$$

for $h=1, \ldots, m-1$, which is (A.23).

Our reals $Q \in \mathcal{S}$ satisfy conditions (A.14), (A.15) of Lemma A.5. Since we assumed that alternative (i) of Lemma A.8 is false, alternative (ii) of Lemma A.5 must also be false. So (i) of that lemma must be true. This implies in particular that

$$
H_{2}\left(\mathbf{x}_{h}\right)>Q_{h}^{\delta / 2\left(\begin{array}{c}
r \\
2
\end{array}\right)} \quad(h=1, \ldots, m) .
$$

Combining this with (A.18), (A.32) implies

$$
\begin{aligned}
& H_{2}\left(\mathbf{x}_{h}\right)^{r_{h}} \geq\left(Q_{h}^{r_{h}}\right)^{\delta / 3}\left(\begin{array}{c}
r \\
2
\end{array}\right) \geq\left(Q_{1}^{r_{1}}\right)^{\delta / 3\left(\begin{array}{c}
r \\
2
\end{array}\right)} \geq C^{r_{1} \delta / 3\left(\begin{array}{c}
r \\
2
\end{array}\right)} \\
& \geq(36 \mathcal{H})^{m r_{1}\left(3 m^{2} / \theta\right)^{m}} \geq\left(e^{q} H_{2}(P)\right)^{\left(3 m^{2} / \theta\right)^{m}}
\end{aligned}
$$

for $h=1, \ldots, m$, which is (A.25). This completes our proof.

Proof of Proposition A.1. First suppose that alternative (ii) of Lemma A.8 is true. By applying Lemma A.4 with $A=Q_{h}, B=Q_{h}^{162 m^{2} / \delta}$ for $h=1, \ldots, m-1$ we conclude that there are one-dimensional linear subspaces $T_{1}, \ldots, T_{t_{4}}$ of $\overline{\mathbb{Q}}^{2}$ with

$$
t_{4} \leq(m-1)\left\{1+\frac{\log \left(162 m^{2} / \delta\right)}{\log (1+\delta / 2)}\right\} \leq 5 \delta^{-1} m \log \left(162 m^{2} / \delta\right)
$$

such that for every $Q$ with

$$
Q \geq C:=(36 \mathcal{H})^{\left(240 m^{2} / \delta\right)^{m} 3 m\left(\begin{array}{c}
r \\
2
\end{array}\right) / \delta}
$$

there is $T_{i} \in\left\{T_{1}, \ldots, T_{t_{4}}\right\}$ with $\left\{\mathbf{x} \in \overline{\mathbb{Q}}^{2}: H_{Q}(\mathbf{x}) \leq Q^{-\delta}\right\} \subset T_{i}$. This also holds true trivially if alternative (i) of Lemma A.8 is true; so it holds true in all cases.

It remains to consider those values $Q$ with

$$
\max \left(\mathcal{H}^{\left.1 /\left(\begin{array}{c}
r \\
2
\end{array}\right), 4^{1 / \delta}\right)=: C^{\prime}<Q<C .}\right.
$$

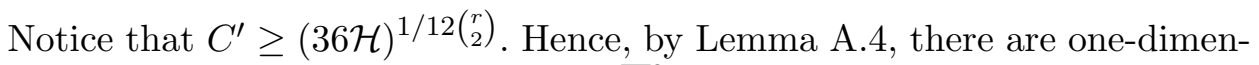
sional linear subspaces $T_{1}^{\prime}, \ldots, T_{t_{5}}^{\prime}$ of $\overline{\mathbb{Q}}^{2}$ with

$$
\begin{aligned}
t_{5} & \leq 1+\frac{\log \left(\log C / \log C^{\prime}\right)}{\log (1+\delta / 2)} \\
& \leq 5 \delta^{-1} m \log \left(240 m^{2} / \delta\right)+\log \left(3 m\left(\begin{array}{l}
r \\
2
\end{array}\right)\right)+\log \left(12\left(\begin{array}{l}
r \\
2
\end{array}\right)\right) \\
& \leq 6 \delta^{-1} m \log \left(240 m^{2} / \delta\right)
\end{aligned}
$$


such that for every $Q$ with (A.33) there is $T_{i}^{\prime} \in\left\{T_{1}^{\prime}, \ldots, T_{t_{5}}^{\prime}\right\}$ with $\left\{\mathbf{x} \in \overline{\mathbb{Q}}^{2}\right.$ : $\left.H_{Q}(\mathbf{x}) \leq Q^{-\delta}\right\} \subset T_{i}^{\prime}$.

Collecting the above, we find that there are one-dimensional linear subspaces $T_{1}, \ldots, T_{t_{2}}$ of $\overline{\mathbb{Q}}^{2}$ with

$$
t_{2} \leq t_{4}+t_{5} \leq 11 \delta^{-1} m \log \left(240 m^{2} / \delta\right) \leq 33 \delta^{-1} m \log m
$$

such that for every $Q>C^{\prime}$ there is $T_{i} \in\left\{T_{1}, \ldots, T_{t_{2}}\right\}$ with $\left\{\mathbf{x} \in \overline{\mathbb{Q}}^{2}\right.$ : $\left.H_{Q}(\mathbf{x}) \leq Q^{-\delta}\right\} \subset T_{i}$. Substituting (A.26) for $m$ we obtain

$$
\begin{aligned}
t_{2} & \leq 33 \delta^{-1} \cdot 25601 \delta^{-2} \log (2 r) \log \left(25601 \delta^{-2} \log (2 r)\right) \\
& <2^{25} \delta^{-3} \log (2 r) \log \left(\delta^{-1} \log (2 r)\right),
\end{aligned}
$$

which is the right-hand side of (A.11).

To finish the proof of Proposition A.1, it remains to show that the spaces $T_{1}, \ldots, T_{t_{2}}$ are defined over $\mathbb{K}$. Let $Q$ be any real $\geq 1$. Suppose that there are non-zero vectors $\mathbf{x} \in \overline{\mathbb{Q}}^{2}$ with $H_{Q}(\mathbf{x}) \leq Q^{-\delta}$, and that these vectors span a one-dimensional linear subspace $T$ of $\overline{\mathbb{Q}}^{2}$. According to [14, Lemma 4.1, p. 32], for any $K$-automorphism $\sigma$ of $\overline{\mathbb{Q}}$ we have $H_{Q}(\sigma(\mathbf{x}))=H_{Q}(\mathbf{x})$, where $\sigma(\mathbf{x})$ is obtained by applying $\sigma$ to the coordinates of $\mathbf{x}$; hence $\sigma(\mathbf{x}) \in T$. This implies that $T$ is defined over $\mathbb{K}$.

\section{References}

[1] B. Adamczewski and Y. Bugeaud, On the complexity of algebraic numbers I. Expansions in integer bases, Ann. of Math. 165 (2007), 547-565.

[2] B. Adamczewski, Y. Bugeaud et F. Luca, Sur la complexité des nombres algébriques, C. R. Math. Acad. Sci. Paris 339 (2004), 11-14.

[3] J. Albert, Propriétés combinatoires et arithmétiques de certaines suites automatiques et substitutives, Thèse de doctorat, Université Paris XI, 2006.

[4] J.-P. Allouche, Nouveaux résultats de transcendance de réels à développements non aléatoires, Gaz. Math. 84 (2000), 19-34.

[5] D. H. Bailey, J. M. Borwein, R. E. Crandall and C. Pomerance, On the binary expansions of algebraic numbers, J. Théor. Nombres Bordeaux 16 (2004), 487-518.

[6] E. Bombieri and W. Gubler, Heights in Diophantine Geometry, New Math. Monogr. 4, Cambridge Univ. Press, 2006.

[7] É. Borel, Sur les chiffres décimaux de $\sqrt{2}$ et divers problèmes de probabilités en chaîne, C. R. Acad. Sci. Paris 230 (1950), 591-593.

[8] Y. Bugeaud, On the b-ary expansion of an algebraic number, Rend. Sem. Mat. Univ. Padova 118 (2007), 217-233.

[9] - Extensions of the Cugiani-Mahler theorem, Ann. Scuola Norm. Sup. Pisa 6 (2007), 477-498.

[10] D. G. Champernowne, The construction of decimals normal in the scale of ten, J. London Math. Soc. 8 (1933), 254-260.

[11] M. Cugiani, Sull'approssimazione di numeri algebrici mediante razionali, Collectanea Mathematica, Pubblicazioni dell'Istituto di Matematica dell'Università di Milano 169, Ed. C. Tanburini, Milano, pagg. 5 (1958). 
[12] J.-H. Evertse, An explicit version of Faltings' Product Theorem and an improvement of Roth's lemma, Acta Arith. 73 (1995), 215-248.

[13] —, An improvement of the quantitative subspace theorem, Compos. Math. 101 (1996), $225-311$.

[14] J.-H. Evertse and H. P. Schlickewei, A quantitative version of the absolute subspace theorem, J. Reine Angew. Math. 548 (2002), 21-127.

[15] S. Ferenczi and Ch. Mauduit, Transcendence of numbers with a low complexity expansion, J. Number Theory 67 (1997), 146-161.

[16] H. Locher, On the number of good approximations of algebraic numbers by algebraic numbers of bounded degree, Acta Arith. 89 (1999), 97-122.

[17] K. Mahler, Lectures on Diophantine Approximation, Part 1: g-adic Numbers and Roth's Theorem, University of Notre Dame, Ann Arbor, 1961.

[18] M. Morse and G. A. Hedlund, Symbolic dynamics, Amer. J. Math. 60 (1938), 815866.

[19] —, -, Symbolic dynamics II, ibid. 62 (1940), 1-42.

[20] J.-J. Pansiot, Bornes inférieures sur la complexité des facteurs des mots infinis engendrés par morphismes itérés, in: STACS 84 (Paris, 1984), Lecture Notes in Comput. Sci. 166, Springer, Berlin, 1984, 230-240.

[21] D. Ridout, Rational approximations to algebraic numbers, Mathematika 4 (1957), 125-131.

[22] T. Rivoal, On the bits counting function of real numbers, J. Austral. Math. Soc., to appear.

[23] K. F. Roth, Rational approximations to algebraic numbers, Mathematika 2 (1955), 1-20; corrigendum, ibid., 168.

[24] W. M. Schmidt, Diophantine Approximation, Lecture Notes in Math. 785, Springer, 1980.

[25] Th. Schneider, Einführung in die transzendenten Zahlen, Springer, Berlin, 1957.

U. F. R. de mathématiques

Université Louis Pasteur

7, rue René Descartes

67084 Strasbourg Cedex, France

E-mail: bugeaud@math.u-strasbg.fr
Mathematisch Instituut Universiteit Leiden Postbus 9512 2300 RA Leiden, The Netherlands E-mail: evertse@math.leidenuniv.nl 